\title{
A robust Khintchine inequality, and algorithms for computing optimal constants in Fourier analysis and high-dimensional geometry
}

\author{
Anindya De* \\ University of California, Berkeley \\ anindya@cs.berkeley.edu \\ Ilias Diakonikolas ${ }^{\dagger}$ \\ University of Edinburgh, Edinburgh, UK \\ ilias.d@ed.ac.uk \\ Rocco A. Servedio $\ddagger$ \\ Columbia University \\ rocco@cs.columbia.edu
}

October 16, 2018

\begin{abstract}
This paper makes two contributions towards determining some well-studied optimal constants in Fourier analysis of Boolean functions and high-dimensional geometry.

1. It has been known since 1994 [GL94] that every linear threshold function has squared Fourier mass at least $1 / 2$ on its degree- 0 and degree- 1 coefficients. Denote the minimum such Fourier mass by $\mathbf{W}^{\leq 1}[\mathbf{L T F}]$, where the minimum is taken over all $n$-variable linear threshold functions and all $n \geq 0$. Benjamini, Kalai and Schramm [BKS99] have conjectured that the true value of $\mathbf{W} \leq 1[\mathbf{L T F}]$ is $2 / \pi$. We make progress on this conjecture by proving that $\mathbf{W} \leq 1[\mathbf{L T F}] \geq 1 / 2+c$ for some absolute constant $c>0$. The key ingredient in our proof is a "robust" version of the well-known Khintchine inequality in functional analysis, which we believe may be of independent interest.

2. We give an algorithm with the following property: given any $\eta>0$, the algorithm runs in time $2^{\text {poly }(1 / \eta)}$ and determines the value of $\mathbf{W}^{\leq 1}[\mathbf{L T F}]$ up to an additive error of $\pm \eta$. We give a similar $2^{\text {poly }(1 / \eta)}$-time algorithm to determine Tomaszewski's constant to within an additive error of $\pm \eta$; this is the minimum (over all origin-centered hyperplanes $H$ ) fraction of points in $\{-1,1\}^{n}$ that lie within Euclidean distance 1 of $H$. Tomaszewski's constant is conjectured to be $1 / 2$; lower bounds on it have been given by Holzman and Kleitman [HK92] and independently by Ben-Tal, Nemirovski and Roos [BTNR02]. Our algorithms combine tools from anti-concentration of sums of independent random variables, Fourier analysis, and Hermite analysis of linear threshold functions.
\end{abstract}

\footnotetext{
${ }^{*}$ Research supported by NSF award CCF-0915929 and NSF award CCF-1017403.

${ }^{\dagger}$ Research performed in part while supported by a Simons Postdoctoral Fellowship at UC Berkeley.

${ }^{\ddagger}$ Supported by NSF grants CCF-0915929 and CCF-1115703.
} 


\section{Introduction}

This paper is inspired by a belief that simple mathematical objects should be well understood. We study two closely related kinds of simple objects: $n$-dimensional linear threshold functions $f(x)=\operatorname{sign}(w$. $x-\theta$ ), and $n$-dimensional origin-centered hyperplanes $H=\left\{x \in \mathbb{R}^{n}: w \cdot x=0\right\}$. Benjamini, Kalai and Schramm [BKS99] and Tomaszewski [Guy86] have posed the question of determining two universal constants related to halfspaces and origin-centered hyperplanes respectively; we refer to these quantities as "the BKS constant" and "Tomaszewski's constant." While these constants arise in various contexts including uniform-distribution learning and optimization theory, little progress has been made on determining their actual values over the past twenty years. In both cases there is an easy upper bound which is conjectured to be the correct value; Gotsman and Linial [GL94] gave the best previously known lower bound on the BKS constant in 1994, and Holzmann and Kleitman [HK92] gave the best known lower bound on Tomaszewski's constant in 1992.

We give two main results. The first of these is an improved lower bound on the BKS constant; a key ingredient in the proof is a "robust" version of the well-known Khintchine inequality, which we believe may be of independent interest. Our second main result is a pair of algorithms for computing the BKS constant and Tomaszewski's constant up to any prescribed accuracy. The first algorithm, given any $\eta>0$, runs in time $2^{\text {poly }(1 / \eta)}$ and computes the BKS constant up to an additive $\eta$, and the second algorithm runs in time $2^{\text {poly }(1 / \eta)}$ and has the same performance guarantee for Tomaszewski's constant.

\subsection{Background and problem statements}

First problem: low-degree Fourier weight of linear threshold functions. A linear threshold function, henceforth denoted simply LTF, is a function $f:\{-1,1\}^{n} \rightarrow\{-1,1\}$ of the form $f(x)=\operatorname{sign}(w \cdot x-\theta)$ where $w \in \mathbb{R}^{n}$ and $\theta \in \mathbb{R}$ (the univariate function $\operatorname{sign}: \mathbb{R} \rightarrow \mathbb{R}$ is $\operatorname{sign}(z)=1$ for $z \geq 0$ and $\operatorname{sign}(z)=-1$ for $z<0)$. The values $w_{1}, \ldots, w_{n}$ are the weights and $\theta$ is the threshold. Linear threshold functions play a central role in many areas of computer science such as concrete complexity theory and machine learning, see e.g. $\left[\mathrm{DGJ}^{+} 10\right]$ and the references therein.

It is well known [BKS99, Per04] that LTFs are highly noise-stable, and hence they must have a large amount of Fourier weight at low degrees. For $f:\{-1,1\}^{n} \rightarrow \mathbb{R}$ and $k \in[0, n]$ let us define $\mathbf{W}^{k}[f]=$ $\sum_{S \subseteq[n],|S|=k} \widehat{f}^{2}(S)$ and $\mathbf{W} \leq k[f]=\sum_{j=0}^{k} \mathbf{W}^{j}[f]$; we will be particularly interested in the Fourier weight of LTFs at levels 0 and 1. More precisely, for $n \in \mathbb{N}$ let $\mathbf{L T F}$ denote the set of all $n$-dimensional LTFs, and let $\mathbf{L T F}=\cup_{n=1}^{\infty} \mathbf{L T F}_{n}$. We define the following universal constant:

Definition 1. $\mathbf{W} \leq 1[\mathbf{L T F}] \stackrel{\text { def }}{=} \inf _{h \in \mathbf{L T F}} \mathbf{W} \leq 1(h)=\inf _{n \in \mathbb{N}} \mathbf{W} \leq 1\left[\mathbf{L T F}_{n}\right]$, where $\mathbf{W}^{\leq 1}\left[\mathbf{L T F}_{n}\right] \stackrel{\text { def }}{=} \inf _{h \in \mathbf{L T F}_{n}} \mathbf{W}^{\leq 1}(h)$.

Benjamini, Kalai and Schramm (see [BKS99], Remark 3.7) and subsequently O'Donnell (see the Conjecture following Theorem 2 of Section 5.1 of [O'D12]) have conjectured that $\mathbf{W}^{\leq 1}[\mathbf{L T F}]=2 / \pi$, and hence we will sometimes refer to $\mathbf{W}^{\leq 1}[\mathbf{L T F}]$ as "the BKS constant." As $n \rightarrow \infty$, a standard analysis of the $n$-variable Majority function shows that $\mathbf{W}^{\leq 1}[\mathbf{L T F}] \leq 2 / \pi$. Gotsman and Linial [GL94] observed that $\mathbf{W}^{\leq 1}[\mathbf{L T F}] \geq 1 / 2$ but until now no better lower bound was known. We note that since the universal constant $\mathbf{W} \leq 1[\mathbf{L T F}]$ is obtained by taking the infimum over an infinite set, it is not a priori clear whether the computational problem of computing or even approximating $\mathbf{W} \leq 1[\mathbf{L T F}]$ is decidable.

Jackson [Jac06] has shown that improved lower bounds on $\mathbf{W}^{\leq 1}[\mathbf{L T F}]$ translate directly into improved noise-tolerance bounds for agnostic weak learning of LTFs in the "Restricted Focus of Attention" model of Ben-David and Dichterman [BDD98]. Further motivation for studying $\mathbf{W}^{\leq 1}[f]$ comes from the fact that 
$\mathbf{W}^{1}[f]$ is closely related to the noise stability of $f$ (see [0'D12]). In particular, if $\mathbf{N S}_{\rho}[f]$ represents the noise stability of $f$ when the noise rate is $(1-\rho) / 2$, then it is known that

$$
\left.\frac{d \mathbf{N S}_{\rho}[f]}{d \rho}\right|_{\rho=0}=\mathbf{W}^{1}[f] .
$$

This means that for a function $f$ with $\mathbf{E}[f]=0$, we have $\mathbf{N S}_{\rho}[f] \rightarrow \rho \cdot \mathbf{W}^{\leq 1}[f]$ as $\rho \rightarrow 0$. Thus, at very large noise rates, $\mathbf{W}^{1}[f]$ quantifies the size of the "noisy boundary" of the mean-zero function $f$.

Second problem: how many hypercube points have distance at most 1 from an origin-centered hyperplane? For $n \in \mathbb{N}$ and $n>1$, let $\mathbb{S}^{n-1}$ denote the $n$-dimensional sphere $\mathbb{S}^{n-1}=\left\{w \in \mathbb{R}^{n}:\|w\|_{2}=1\right\}$, and let $\mathbb{S}=\cup_{n>1} \mathbb{S}^{n-1}$. Each unit vector $w \in \mathbb{S}^{n-1}$ defines an origin-centered hyperplane $H_{w}=\left\{x \in \mathbb{R}^{n}\right.$ : $w \cdot x=0\}$. Given a unit vector $w \in \mathbb{S}^{n-1}$, we define $\mathbf{T}(w) \in[0,1]$ to be $\mathbf{T}(w)=\mathbf{P r}_{x \in\{-1,1\}^{n}}[|w \cdot x| \leq 1]$, the fraction of hypercube points in $\{-1,1\}^{n}$ that lie within Euclidean distance 1 of the hyperplane $H_{w}$. We define the following universal constant, which we call "Tomaszewski's constant:"

Definition 2. $\mathbf{T}(\mathbb{S}) \stackrel{\text { def }}{=} \inf _{w \in \mathbb{S}} \mathbf{T}(w)=\inf _{n \in \mathbb{N}} \mathbf{T}\left(\mathbb{S}^{n-1}\right)$, where $\mathbf{T}\left(\mathbb{S}^{n-1}\right) \stackrel{\text { def }}{=} \inf _{w \in \mathbb{S}^{n-1}} \mathbf{T}(w)$.

Tomaszewski [Guy86] has conjectured that $\mathbf{T}(\mathbb{S})=1 / 2$. The main result of Holzman and Kleitman [HK92] is a proof that $3 / 8 \leq \mathbf{T}(\mathbb{S})$; the upper bound $\mathbf{T}(\mathbb{S}) \leq 1 / 2$ is witnessed by the vector $w=(1 / \sqrt{2}, 1 / \sqrt{2})$. As noted in [HK92], the quantity $\mathbf{T}(\mathbb{S})$ has a number of appealing geometric and probabilistic reformulations. Similar to the BKS constant, since $\mathbf{T}(\mathbb{S})$ is obtained by taking the infimum over an infinite set, it is not immediately evident that any algorithm can compute or approximate $\mathbf{T}(\mathbb{S})$. 1 .

An interesting quantity in its own right, Tomaszewski's constant also arises in a range of contexts in optimization theory, see e.g. [So09, BTNR02]. In fact, the latter paper proves a lower bound of $1 / 3$ on the value of Tomaszewski's constant independently of [HK92], and independently conjectures that the optimal lower bound is $1 / 2$.

\subsection{Our results}

A better lower bound for the BKS constant $\mathbf{W}^{\leq 1}[\mathbf{L T F}]$. Our first main result is the following theorem:

Theorem 3 (Lower Bound for the BKS constant). There exists a universal constant $c^{\prime}>0$ such that $\mathbf{W} \leq 1[\mathbf{L T F}] \geq \frac{1}{2}+c^{\prime}$.

This is the first improvement on the [GL94] lower bound of $1 / 2$ since 1994. We actually give two quite different proofs of this theorem, which are sketched in the "Techniques" subsection below.

An algorithm for approximating the BKS constant $\mathbf{W}^{\leq 1}[\mathbf{L T F}]$. Our next main result shows that in fact there is a finite-time algorithm that approximates the BKS constant up to any desired accuracy:

Theorem 4 (Approximating the BKS constant). There is an algorithm that, on input an accuracy parameter $\epsilon>0$, runs in time $2^{\mathrm{poly}(1 / \epsilon)}$ and outputs a value $\Gamma_{\epsilon}$ such that

$$
\mathbf{W}^{\leq 1}[\mathbf{L T F}] \leq \Gamma_{\epsilon} \leq \mathbf{W}^{\leq 1}[\mathbf{L T F}]+\epsilon .
$$

An algorithm for approximating Tomaszewski's constant $\mathbf{T}(\mathbb{S})$. Our final main result is a similar-in-spirit algorithm that approximates $\mathbf{T}(\mathbb{S})$ up to any desired accuracy:

Theorem 5 (Approximating Tomaszewski's constant). There is an algorithm that, on input an accuracy parameter $\epsilon>0$, runs in time $2^{\text {poly }(1 / \epsilon)}$ and outputs a value $\Gamma_{\epsilon}$ such that

$$
\mathbf{T}(\mathbb{S}) \leq \Gamma_{\epsilon} \leq \mathbf{T}(\mathbb{S})+\epsilon .
$$

\footnotetext{
${ }^{1}$ Whenever we speak of "an algorithm to compute or approximate" one of these constants, of course what we really mean is an algorithm that outputs the desired value together with a proof of correctness of its output value.
} 


\subsection{Our techniques for Theorem 3; lower-bounding the BKS constant $\mathrm{W}^{\leq 1}[\mathrm{LTF}]$}

It is easy to show that it suffices to consider the level-1 Fourier weight $\mathbf{W}^{1}$ of LTFs that have threshold $\theta=0$ and have $w \cdot x \neq 0$ for all $x \in\{-1,1\}^{n}$, so we confine our discussion to such zero-threshold LTFs (see Fact 39 for a proof). To explain our approaches to lower bounding $\mathbf{W} \leq 1$ [LTF], we recall the essentials of Gotsman and Linial's simple argument that gives a lower bound of $1 / 2$. The key ingredient of their argument is the well-known Khintchine inequality from functional analysis:

Definition 6. For a unit vector $w \in \mathbb{S}^{n-1}$ we define

$$
\mathbf{K}(w) \stackrel{\text { def }}{=} \mathbf{E}_{x \in\{-1,1\}^{n}}[|w \cdot x|]
$$

to be the "Khintchine constant for w."

The following is a classical theorem in functional analysis (we write $e_{i}$ to denote the unit vector in $\mathbb{R}^{n}$ with a 1 in coordinate $i$ ):

Theorem 7 (Khintchine inequality, [Sza76]). For $w \in \mathbb{S}^{n-1}$ any unit vector, we have $\mathbf{K}(w) \geq 1 / \sqrt{2}$, with equality holding if and only if $w=\frac{1}{\sqrt{2}}\left( \pm e_{i} \pm e_{j}\right)$ for some $i \neq j \in[n]$.

Szarek [Sza76] was the first to obtain the optimal constant $1 / \sqrt{2}$, and subsequently several simplifications of his proof were given [Haa82, Tom87, LO94]; we shall give a simple self-contained proof in Section 3.1 below. This proof has previously appeared in [Gar07, Fil12] and is essentially a translation of the [LO94] proof into "Fourier language." With Theorem 7 in hand, the Gotsman-Linial lower bound is almost immediate:

Proposition 8 ([GL94]). Let $f:\{-1,1\}^{n} \rightarrow\{-1,1\}$ be a zero-threshold LTF $f(x)=\operatorname{sign}(w \cdot x)$ where $w \in \mathbb{R}^{n}$ has $\|w\|_{2}=1$. Then $\mathbf{W}^{1}[f] \geq(\mathbf{K}(w))^{2}$.

Proof. We have that

$$
\mathbf{K}(w)=\mathbf{E}_{x}[f(x)(w \cdot x)]=\sum_{i=1}^{n} \widehat{f}(i) w_{i} \leq \sqrt{\sum_{i=1}^{n} \widehat{f}^{2}(i)} \cdot \sqrt{\sum_{i=1}^{n} w_{i}^{2}}=\sqrt{\mathbf{W}^{1}[f]}
$$

where the first equality uses the definition of $f$, the second is Plancherel's identity, the inequality is CauchySchwarz, and the last equality uses the assumption that $w$ is a unit vector.

First proof of Theorem 3: A "robust" Khintchine inequality. Given the strict condition required for equality in the Khintchine inequality, it is natural to expect that if a unit vector $w \in \mathbb{R}^{n}$ is "far" from $\frac{1}{\sqrt{2}}\left( \pm e_{i} \pm e_{j}\right)$, then $\mathbf{K}(w)$ should be significantly larger than $1 / \sqrt{2}$. We prove a robust version of the Khintchine inequality which makes this intuition precise. Given a unit vector $w \in \mathbb{S}^{n-1}$, define $d(w)$ to be $d(w)=\min \left\|w-w^{*}\right\|_{2}$, where $w^{*}$ ranges over all $4\left(\begin{array}{l}n \\ 2\end{array}\right)$ vectors of the form $\frac{1}{\sqrt{2}}\left( \pm e_{i} \pm e_{j}\right)$. Our "robust Khintchine" inequality is the following:

Theorem 9 (Robust Khintchine inequality). There exists a universal constant $c>0$ such that for any $w \in \mathbb{S}^{n-1}$, we have

$$
\mathbf{K}(w) \geq \frac{1}{\sqrt{2}}+c \cdot d(w)
$$


Armed with our robust Khintchine inequality, the simple proof of Proposition 8 suggests a natural approach to lower-bounding $\mathbf{W} \leq 1[\mathbf{L T F}]$. If $w$ is such that $d(w)$ is "large" (at least some absolute constant), then the statement of Proposition 8 immediately gives a lower bound better than $1 / 2$. So the only remaining vectors $w$ to handle are highly constrained vectors which are almost exactly of the form $\frac{1}{\sqrt{2}}\left( \pm e_{i} \pm e_{j}\right)$. A natural hope is that the Cauchy-Schwarz inequality in the proof of Proposition 8 is not tight for such highly constrained vectors, and indeed this is essentially how we proceed (modulo some simple cases in which it is easy to bound $\mathbf{W}^{\leq 1}$ above $1 / 2$ directly).

Second proof of Theorem 3: anticoncentration, Fourier analysis of LTFs, and LTF approximation. Our second proof of Theorem 3 employs several sophisticated ingredients from recent work on structural properties of LTFs [OS11, MORS10]. The first of these ingredients is a result (Theorem 6.1 of [OS11]) which essentially says that any LTF $f(x)=\operatorname{sign}(w \cdot x)$ can be perturbed very slightly to another LTF $f^{\prime}(x)=\operatorname{sign}\left(w^{\prime} \cdot x\right)$ (where both $w$ and $w^{\prime}$ are unit vectors). The key properties of this perturbation are that (i) $f$ and $f^{\prime}$ are extremely close, differing only on a tiny fraction of inputs in $\{-1,1\}^{n}$; but (ii) the linear form $w^{\prime} \cdot x$ has some nontrivial "anti-concentration" when $x$ is distributed uniformly over $\{-1,1\}^{n}$, meaning that very few inputs have $w^{\prime} \cdot x$ very close to 0 .

Why is this useful? It turns out that the anti-concentration of $w^{\prime} \cdot x$, together with results on the degree-1 Fourier spectrum of "regular" halfspaces from [MORS10], lets us establish a lower bound on $\mathbf{W} \leq 1\left[f^{\prime}\right]$ that is strictly greater than $1 / 2$. Then the fact that $f$ and $f^{\prime}$ agree on almost every input in $\{-1,1\}^{n}$ lets us argue that the original LTF $f$ must similarly have $\mathbf{W} \leq 1[f]$ strictly greater than $1 / 2$. Interestingly, the lower bound on $\mathbf{W} \leq 1\left[f^{\prime}\right]$ is proved using the Gotsman-Linial inequality $\mathbf{W} \leq 1\left[f^{\prime}\right] \geq\left(\mathbf{K}\left(w^{\prime}\right)\right)^{2}$; in fact, the anticoncentration of $w^{\prime} \cdot x$ is combined with ingredients in the simple Fourier proof of the (original, non-robust) Khintchine inequality (specifically, an upper bound on the total influence of the function $\ell(x)=\left|w^{\prime} \cdot x\right|$ ) to obtain the result.

\subsection{Our techniques for Theorem 4: approximating the BKS constant $\mathrm{W}^{\leq 1}[\mathrm{LTF}]$}

As in the previous subsection, it suffices to consider only zero-threshold LTFs $\operatorname{sign}(w \cdot x)$. Our algorithm turns out to be very simple (though its analysis is not):

Let $K=\Theta\left(\epsilon^{-24}\right)$. Enumerate all $K$-variable zero-threshold LTFs, and output the value

$$
\Gamma_{\epsilon} \stackrel{\text { def }}{=} \min \left\{\mathbf{W}^{1}[f]: f \text { is a zero-threshold } K \text {-variable LTF. }\right\} \text {. }
$$

It is well known (see e.g. [MT94]) that there exist $2^{\Theta\left(K^{2}\right)}$ distinct $K$-variable LTFs, and it is straightforward to confirm that they can be enumerated in time $2^{O\left(K^{2} \log K\right)}$. Since $\mathbf{W}^{1}[f]$ can be computed in time $2^{O(K)}$ for any given $K$-variable LTF $f$, the above simple algorithm runs in time $2^{\text {poly }(1 / \epsilon)}$; the challenge is to show that the value $\Gamma_{\epsilon}$ thus obtained indeed satisfies Equation (1).

A key ingredient in our analysis is the notion of the "critical index" of an LTF $f$. The critical index was implicitly introduced and used in [Ser07] and was explicitly used in [DS09, DGJ $^{+} 10$, OS11, DDFS12] and other works. To define the critical index we need to first define "regularity":

Definition 10 (regularity). Fix any real value $\tau>0$. We say that a vector $w=\left(w_{1}, \ldots, w_{n}\right) \in \mathbb{R}^{n}$ is $\tau$-regular if $\max _{i \in[n]}\left|w_{i}\right| \leq \tau\|w\|=\tau \sqrt{w_{1}^{2}+\cdots+w_{n}^{2}}$. A linear form $w \cdot x$ is said to be $\tau$-regular if $w$ is $\tau$-regular, and similarly an LTF is said to be $\tau$-regular if it is of the form $\operatorname{sign}(w \cdot x-\theta)$ where $w$ is $\tau$-regular.

Regularity is a helpful notion because if $w$ is $\tau$-regular then the Berry-Esséen theorem tells us that for uniform $x \in\{-1,1\}^{n}$, the linear form $w \cdot x$ is "distributed like a Gaussian up to error $\tau$." This can be useful for many reasons (as we will see below). 
Intuitively, the critical index of $w$ is the first index $i$ such that from that point on, the vector $\left(w_{i}, w_{i+1}, \ldots, w_{n}\right)$ is regular. A precise definition follows:

Definition 11 (critical index). Given a vector $w \in \mathbb{R}^{n}$ such that $\left|w_{1}\right| \geq \cdots \geq\left|w_{n}\right|>0$, for $k \in[n]$ we denote by $\sigma_{k}$ the quantity $\sqrt{\sum_{i=k}^{n} w_{i}^{2}}$. We define the $\tau$-critical index $c(w, \tau)$ of $w$ as the smallest index $i \in[n]$ for which $\left|w_{i}\right| \leq \tau \cdot \sigma_{i}$. If this inequality does not hold for any $i \in[n]$, we define $c(w, \tau)=\infty$.

Returning to Theorem 4, since our algorithm minimizes over a proper subset of all LTFs, it suffices to show that for any zero-threshold LTF $f=\operatorname{sign}(w \cdot x)$, there is a $K$-variable zero-threshold LTF $g$ such that

$$
\mathbf{W}^{1}[g]-\mathbf{W}^{1}[f]<\epsilon .
$$

At a high level our proof is a case analysis based on the size of the $\delta$-critical index $c(w, \delta)$ of the weight vector $w$, where we choose the parameter $\delta$ to be $\delta=\operatorname{poly}(\epsilon)$. The first case is relatively easy: if the $\delta$-critical index is large, then it is known that the function $f$ is very close to some $K$-variable LTF $g$. Since the two functions agree almost everywhere, it is easy to show that $\left|\mathbf{W}^{1}[f]-\mathbf{W}^{1}[g]\right| \leq \epsilon$ as desired.

The case that the critical index is small is much more challenging. In this case it is by no means true that $f$ can be well approximated by an LTF on few variables - consider, for example, the majority function. We deal with this challenge by developing a novel variable reduction technique which lets us construct a poly $(1 / \epsilon)$-variable LTF $g$ whose level-1 Fourier weight closely matches that of $f$.

How is this done? The answer again comes from the critical index. Since the critical index $c(w, \delta)$ is small, we know that except for the "head" portion $\sum_{i=1}^{c(w, \delta)-1} w_{i} x_{i}$ of the linear form, the "tail" portion $\sum_{i=c(w, \delta)}^{n} w_{i} x_{i}$ of the linear form "behaves like a Gaussian." Guided by this intuition, our variable reduction technique proceeds in three steps. In the first step, we replace the tail coordinates $x_{T}=\left(x_{c(w, \delta)}, \ldots, x_{n}\right)$ by independent Gaussian random variables and show that the degree-1 Fourier weight of the corresponding "mixed" function (which has some \pm 1 -valued inputs and some Gaussian inputs) is approximately equal to $\mathbf{W}^{1}[f]$. In the second step, we replace the tail random variable $w_{T} \cdot G_{T}$, where $G_{T}$ is the vector of Gaussians from the first step, by a single Gaussian random variable $G$, where $G \sim \mathcal{N}\left(0,\left\|w_{T}\right\|^{2}\right)$. We show that this transformation exactly preserves the degree- 1 weight. At this point we have reduced the number of variables from $n$ down to $c(w, \delta)$ (which is small in this case!), but the last variable is Gaussian rather than Boolean. As suggested by the Central Limit Theorem, though, one may try to replace this Gaussian random variable by a normalized sum of independent \pm 1 random variables $\sum_{i=1}^{M} z_{i} / \sqrt{M}$. This is exactly the third step of our variable reduction technique. Via a careful analysis, we show that by taking $M=\operatorname{poly}(1 / \epsilon)$, this operation preserves the degree- 1 weight up to an additive $\epsilon$. Combining all these steps, we obtain the desired result.

\subsection{Our techniques for Theorem 5 ; approximating Tomaszewski's constant $\mathrm{T}(\mathbb{S})$}

The first step of our proof of Theorem 5 is similar in spirit to the main structural ingredient of our proof of Theorem 4. we show (Theorem 69) that given any $\epsilon>0$, there is a value $K_{\epsilon}=\operatorname{poly}(1 / \epsilon)$ such that it suffices to consider linear forms $w \cdot x$ over $K_{\epsilon}$-dimensional space, i.e. for any $n \in \mathbb{N}$ we have

$$
\mathbf{T}\left(\mathbb{S}^{n-1}\right) \leq \mathbf{T}\left(\mathbb{S}^{K_{\epsilon}-1}\right) \leq \mathbf{T}\left(\mathbb{S}^{n-1}\right)+\epsilon
$$

Similar to the high-level outline of Theorem 4 , our proof again proceeds by fixing any $w \in \mathbb{S}^{n-1}$ and doing a case analysis based on whether the critical index of $w$ is "large" or "small." However, the technical details of each of these cases is quite different from the earlier proof. In the "small critical index" case we employ Gaussian anti-concentration (which is inherited by the "tail" random variable $w_{T} x_{T}$ since the tail vector $w_{T}$ is regular), and in the "large critical index" case we use an anti-concentration result from [OS11]. 
Unlike the previous situation for the BKS constant, at this point more work remains to be done for approximating Tomaszewski's constant. While there are only $2^{\text {poly }(1 / \epsilon)}$ many halfspaces over poly $(1 / \epsilon)$ many variables and hence a brute-force enumeration could cover all of them in $2^{\text {poly }(1 / \epsilon)}$ time for the BKS constant, here we must contend with the fact that $\mathbb{S}^{K_{\epsilon}-1}$ is an uncountably infinite set, so we cannot naively minimize over all its elements. Instead we take a dual approach and exploit the fact that while there are uncountably infinitely many vectors in $\mathbb{S}^{K_{\epsilon}-1}$, there are only $2^{K_{\epsilon}}$ many hypercube points in $\{-1,1\}{ }^{K_{\epsilon}}$, and (with some care) the desired infimum over all unit vectors can be formulated in the language of existential theory of the reals. We then use an algorithm for deciding existential theory of the reals (see [Ren88]) to compute the infimum.

Discussion. It is interesting to note that determining Tomaszewski's constant is an instance of the wellstudied generic problem of understanding tails of Rademacher sums. For the sake of discussion, let us define $\mathbf{T}_{\text {in }}(w, a)=\mathbf{P r}_{x \in\{-1,1\}^{n}}[|w \cdot x| \leq a]$ and $\mathbf{T}_{\text {out }}(w, a)=\mathbf{P r}_{x \in\{-1,1\}^{n}}[|w \cdot x| \geq a]$ where $w \in \mathbb{S}^{n-1}$. Further, let $\mathbf{T}_{\text {in }}(a)=\inf _{w \in \mathbb{S}} \mathbf{T}_{\text {in }}(w, a)$ and $\mathbf{T}_{\text {out }}(a)=\inf _{w \in \mathbb{S}} \mathbf{T}_{\text {out }}(w, a)$. Note that Tomaszewski's constant $\mathbf{T}(\mathbb{S})$ is simply $\mathbf{T}_{\text {in }}(1)$. Much effort has been expended on getting sharp estimates for $\mathbf{T}_{\text {in }}(a)$ and $\mathbf{T}_{\text {out }}(a)$ for various values of $a$ (see e.g. [Pin12, Ben04]). As a representative example, Bentkus and Dzindzalieta [BD12] proved that

$$
\mathbf{T}_{\mathrm{in}}(a) \geq \frac{1}{4}+\frac{1}{4} \cdot \sqrt{2-\frac{2}{a^{2}}}
$$

for $a \in(1, \sqrt{2}]$. Similarly, Pinelis [Pin94] showed that there is an absolute constant $c>0$ such that $\mathbf{T}_{\text {out }}(a) \geq 1-c \cdot \frac{\phi(a)}{a}$ where $\phi(x)$ is the density function of the standard normal $\mathcal{N}(0,1)$ (note this beats the standard Hoeffding bound by a factor of $1 / a$ ).

On the complementary side, Montgomery-Smith [MS90] proved that there is an absolute constant $c^{\prime}>0$ such that $\mathbf{T}_{\text {out }}(a) \geq e^{-c^{\prime} \cdot a^{2}}$ for all $a \leq 1$. Similarly, Oleszkiewicz [Ole96] proved that $\mathbf{T}_{\text {out }}(1) \geq 1 / 10$. The conjectured lower bound on $\mathbf{T}_{\text {out }}(1)$ is $7 / 32$ (see [HK94]). While we have not investigated this in detail, we suspect that our techniques may be applicable to some of the above problems. Finally, we note that apart from being of intrinsic interest to functional analysts and probability theorists, the above quantities arise frequently in the optimization literature (see [HLNZ08, BTNR02]). Related tail bounds have also found applications in extremal combinatorics (see [AHS12]).

\section{Mathematical Preliminaries}

\subsection{Fourier analysis over $\{-1,1\}^{n}$ and influences}

We consider functions $f:\{-1,1\}^{n} \rightarrow \mathbb{R}$ (though we often focus on Boolean-valued functions which map to $\{-1,1\}$ ), and we think of the inputs $x$ to $f$ as being distributed according to the uniform probability distribution. The set of such functions forms a $2^{n}$-dimensional inner product space with inner product given by $\langle f, g\rangle=\mathbf{E}_{x}[f(x) g(x)]$. The set of functions $\left(\chi_{S}\right)_{S \subseteq[n]}$ defined by $\chi_{S}(x)=\prod_{i \in S} x_{i}$ forms a complete orthonormal basis for this space. We will also often write simply $x_{S}$ for $\prod_{i \in S} x_{i}$. Given a function $f:\{-1,1\}^{n} \rightarrow \mathbb{R}$ we define its Fourier coefficients by $\widehat{f}(S)=\mathbf{E}_{x}\left[f(x) x_{S}\right]$, and we have that $f(x)=\sum_{S} \widehat{f}(S) x_{S}$.

As an easy consequence of orthonormality we have Plancherel's identity $\langle f, g\rangle=\sum_{S} \widehat{f}(S) \widehat{g}(S)$, which has as a special case Parseval's identity, $\mathbf{E}_{x}\left[f(x)^{2}\right]=\sum_{S} \widehat{f}(S)^{2}$. From this it follows that for every $f:\{-1,1\}^{n} \rightarrow\{-1,1\}$ we have $\sum_{S} \widehat{f}(S)^{2}=1$. Note that for $f:\{-1,1\}^{n} \rightarrow \mathbb{R}$ we have that $\operatorname{Var}[f]=\mathbf{E}_{x}\left[f^{2}(x)\right]-\left(\mathbf{E}_{x}[f]\right)^{2}=\sum_{S \neq \emptyset} \widehat{f}^{2}(S)$.

Definition 12. Given $f:\{-1,1\}^{n} \rightarrow \mathbb{R}$ and $i \in[n]$, the influence of variable $i$ is defined as $\mathbf{I n f}_{i}(f)=$ $\mathbf{E}_{x}\left[\operatorname{Var}_{x_{i}}[f(x)]\right]$. The total influence of $f$ is defined as $\mathbf{I n f}(f)=\sum_{i=1}^{n} \operatorname{Inf}_{i}(f)$. 
Fact 13. We have the identity $\operatorname{Inf}_{i}(f)=\sum_{S \ni i} \widehat{f}^{2}(S)$; moreover, for $f:\{-1,1\}^{n} \rightarrow\{-1,1\}$ (i.e. Boolean-valued), it holds $\operatorname{Inf}_{i}(f)=\operatorname{Pr}_{x}\left[f\left(x^{i-}\right) \neq f\left(x^{i+}\right)\right]$, where $x^{i-}$ and $x^{i+}$ denote $x$ with the i'th bit set to -1 or 1 respectively. If $f:\{-1,1\}^{n} \rightarrow\{-1,1\}$ is unate, then $\operatorname{Inf}_{i}(f)=|\hat{f}(i)|$.

Fact 14. Let $f=\operatorname{sign}\left(\sum_{i=1}^{n} w_{i} x_{i}-w_{0}\right)$ be an LTF such that $\left|w_{1}\right| \geq\left|w_{i}\right|$ for all $i \in[n]$. Then $\left|\operatorname{Inf}_{1}(f)\right| \geq$ $\left|\operatorname{Inf}_{i}(f)\right|$ for all $i \in[n]$. Moreover, for all $i \in[n]$ it holds $w_{i} \cdot \widehat{f}(i) \geq 0$.

\subsection{Probabilistic Facts}

We require some basic probability results including the standard additive Hoeffding bound:

Theorem 15. Let $X_{1}, \ldots, X_{n}$ be independent random variables such that for each $j \in[n], X_{j}$ is supported on $\left[a_{j}, b_{j}\right]$ for some $a_{j}, b_{j} \in \mathbb{R}, a_{j} \leq b_{j}$. Let $X=\sum_{j=1}^{n} X_{j}$. Then, for any $t>0, \operatorname{Pr}[|X-\mathbf{E}[X]| \geq t] \leq$ $2 \exp \left(-2 t^{2} / \sum_{j=1}^{n}\left(b_{j}-a_{j}\right)^{2}\right)$.

The Berry-Esséen theorem (see e.g. [Fel68]) gives explicit error bounds for the Central Limit Theorem:

Theorem 16. (Berry-Esséen) Let $X_{1}, \ldots, X_{n}$ be independent random variables satisfying $\mathbf{E}\left[X_{i}\right]=0$ for all $i \in[n], \sqrt{\sum_{i} \mathbf{E}\left[X_{i}^{2}\right]}=\sigma$, and $\sum_{i} \mathbf{E}\left[\left|X_{i}\right|^{3}\right]=\rho_{3}$. Let $S=\left(X_{1}+\cdots+X_{n}\right) / \sigma$ and let $F$ denote the cumulative distribution function (cdf) of $S$. Then $\sup _{x}|F(x)-\Phi(x)| \leq \rho_{3} / \sigma^{3}$ where $\Phi$ denotes the cdf of the standard gaussian random variable.

An easy consequence of the Berry-Esséen theorem is the following fact, which says that a regular linear form has good anti-concentration (i.e. it assigns small probability mass to any small interval):

Fact 17. Let $w=\left(w_{1}, \ldots, w_{n}\right)$ be a $\tau$-regular vector in $\mathbb{R}^{n}$ and write $\sigma$ to denote $\|w\|_{2}$. Then for any interval $[a, b] \subseteq \mathbb{R}$, we have $\left|\operatorname{Pr}\left[\sum_{i=1}^{n} w_{i} x_{i} \in(a, b]\right]-\Phi([a / \sigma, b / \sigma])\right| \leq 2 \tau$, where $\Phi([c, d]) \stackrel{\text { def }}{=} \Phi(d)-$ $\Phi(c)$. In particular, it follows that $\operatorname{Pr}\left[\sum_{i=1}^{n} w_{i} x_{i} \in(a, b]\right] \leq|b-a| / \sigma+2 \tau$.

\subsection{Technical Tools about Regularity and the Critical Index}

The following simple fact states that the "tail weight" of the vector $w$ decreases exponentially prior to the critical index:

Fact 18. For any vector $w=\left(w_{1}, \ldots, w_{n}\right)$ such that $\left|w_{1}\right| \geq \cdots \geq\left|w_{n}\right|>0$ and $1 \leq a \leq c(w, \tau)$, we have $\sigma_{a}<\left(1-\tau^{2}\right)^{(a-1) / 2} \cdot \sigma_{1}$.

Proof. If $a<c(w, \tau)$, then by definition $\left|w_{a}\right|>\tau \cdot \sigma_{a}$. This implies that $\sigma_{a+1}<\sqrt{1-\tau^{2}} \cdot \sigma_{a}$. Applying this inequality repeatedly, we get that $\sigma_{a}<\left(1-\tau^{2}\right)^{(a-1) / 2} \cdot \sigma_{1}$ for any $1 \leq a \leq c(w, \tau)$.

We will also need the following corollary (that appears e.g. as Propositions 31 and 32 in [MORS10]).

Fact 19. Let $\ell(x)=w \cdot x-w_{0}$ with $\|w\|_{2}=1$ and $w_{0} \in \mathbb{R}$ and $f(x)=\operatorname{sign}(\ell(x))$. If $w$ is $\tau$-regular, then we have:

- (i) $\mathbf{E}_{x \sim \mathcal{U}_{n}}[f(x)] \approx^{\tau} \mathbf{E}_{x \sim \mathcal{N}^{n}}[f(x)]$ and

- (ii) $\mathbf{E}_{x \sim \mathcal{U}_{n}}[|\ell(x)|] \approx^{\tau} \mathbf{E}_{x \sim \mathcal{N} n}[|\ell(x)|]$,

where $\mathcal{N}$ denotes the standard Gaussian distribution $N(0,1)$. 


\subsection{Miscellaneous}

For $a, b \in \mathbb{R}$ we write $a \stackrel{\eta}{\approx} b$ to indicate that $|a-b| \leq O(\eta)$.

For a vector $w \in \mathbb{R}^{n}$, we write $w^{(k)}$ to denote the $(n-k)$-dimensional vector obtained by taking the last $n-k$ coordinates of $w$, i.e. $w^{(k)}=\left(w_{k+1}, \ldots, w_{n}\right)$.

We will use the following elementary fact, which is a direct consequence of Cauchy-Schwarz.

Fact 20. Let $a, b \in \mathbb{R}^{m}$ with $\|a\|_{2} \leq 1$, $\|b\|_{2} \leq 1$ such that $\|a-b\|_{2}^{2} \leq \eta$. Then

$$
\left|\|a\|_{2}^{2}-\|b\|_{2}^{2}\right| \leq 2 \sqrt{\eta}
$$

Proof. We have that

$$
\begin{aligned}
\left|\sum_{i=1}^{m}\left(a_{i}^{2}-b_{i}^{2}\right)\right|=\left|\sum_{i=1}^{m}\left(a_{i}-b_{i}\right)\left(a_{i}+b_{i}\right)\right| & \leq \sqrt{\sum_{i=1}^{m}\left(a_{i}+b_{i}\right)^{2}} \cdot \sqrt{\sum_{i=1}^{m}\left(a_{i}-b_{i}\right)^{2}} \\
& \leq \sqrt{2 \cdot \sum_{i=1}^{m}\left(a_{i}^{2}+b_{i}^{2}\right)} \cdot\|a-b\|_{2} \leq 2 \sqrt{\eta}
\end{aligned}
$$

where the first inequality is Cauchy-Schwarz, the second uses the elementary fact $(a+b)^{2} \leq 2\left(a^{2}+b^{2}\right)$, for all $a, b \in \mathbb{R}$, while the third uses our assumption that $\|a\|_{2},\|b\|_{2} \leq 1$.

\section{Proof of Theorem 9: A "robust" Khintchine inequality}

It will be convenient for us to reformulate Theorems 7 and 9 as follows: Let us say that a unit vector $w=\left(w_{1}, \ldots, w_{n}\right) \in \mathbb{S}^{n-1}$ is proper if $w_{i} \geq w_{i+1} \geq 0$ for all $i \in[n-1]$. Then we may state the "basic" Khintchine inequality with optimal constant, Theorem 7 , in the following equivalent way:

Theorem 21 (Khintchine inequality, [Sza76]). Let $w \in \mathbb{R}^{n}$ be a proper unit vector, so $w_{1} \geq \cdots \geq w_{n} \geq 0$. Then $\mathbf{K}(w) \geq 1 / \sqrt{2}$, with equality holding if and only if $w=w^{*} \stackrel{\text { def }}{=}(1 / \sqrt{2}, 1 / \sqrt{2}, 0, \ldots, 0)$.

And we may restate our "robust" Khintchine inequality, Theorem 9 as follows:

Theorem 22 (Robust Khintchine inequality). There exists a universal constant $c>0$ such that the following holds: Let $w \in \mathbb{R}^{n}$ be a proper unit vector. Then $\mathbf{K}(w) \geq 1 / \sqrt{2}+c \cdot\left\|w-w^{*}\right\|_{2}$, where $w^{*} \stackrel{\text { def }}{=}(1 / \sqrt{2}, 1 / \sqrt{2}, 0, \ldots, 0)$.

Before we proceed with the proof of Theorem 22, we give a simple Fourier analytic proof of the "basic" Khintchine inequality with optimal constant, $\mathbf{K}(w) \geq 1 / \sqrt{2}$. (We note that this is a well-known argument by now; it is given in somewhat more general form in [Ole99] and in [KLO96].) We then build on this to prove Theorem 22

\subsection{Warm-up: simple proof that $\mathbf{K}(w) \geq 1 / \sqrt{2}$}

We consider the function $\ell(x)=\left|\sum_{i=1}^{n} w_{i} x_{i}\right|$ where $\sum_{i} w_{i}^{2}=1$ and will show that $\mathbf{K}(w)=\mathbf{E}_{x}[\ell(x)] \geq$ $1 / \sqrt{2}$. Noting that $\mathbf{E}_{x}\left[(\ell(x))^{2}\right]=1$, we have $(\mathbf{E}[\ell(x)])^{2}=1-\operatorname{Var}[\ell]$, so it suffices to show that $\operatorname{Var}[\ell] \leq$ $1 / 2$. This follows directly by combining the following claims. The first bound is an improved Poincaré inequality for even functions:

Fact 23. (Poincaré inequality) Let $f:\{-1,1\}^{n} \rightarrow \mathbb{R}$ be even. Then $\operatorname{Var}[f] \leq(1 / 2) \cdot \operatorname{Inf}(f)$. 
Proof. Since $f$ is even, we have that $\widehat{f}(S)=0$ for all $S$ with odd $|S|$. We can thus write

$$
\begin{aligned}
\operatorname{Inf}(f)=\sum_{S \subseteq[n],|S| \text { even }}|S| \cdot \widehat{f}^{2}(S) & \geq 2 \cdot \sum_{\emptyset \neq S \subseteq[n],|S| \text { even }} \widehat{f}^{2}(S) \\
& =2 \cdot \sum_{\emptyset \neq S \subseteq[n]} \hat{f}^{2}(S)=2 \cdot \operatorname{Var}[f] .
\end{aligned}
$$

The second is an upper bound on the influences in $\ell$ as a function of the weights:

Lemma 24. Let $\ell(x)=\left|\sum_{i=1}^{n} w_{i} x_{i}\right|$. For any $i \in[n]$, we have $\operatorname{Inf}_{i}(\ell) \leq w_{i}^{2}$.

Proof. Recall that $\operatorname{Inf}_{i}(\ell)=\mathbf{E}_{x}\left[\operatorname{Var}_{x_{i}}[\ell(x)]\right]=\mathbf{E}_{x}\left[\mathbf{E}_{x_{i}}\left[\ell^{2}(x)\right]-\left(\mathbf{E}_{x_{i}}[\ell(x)]\right)^{2}\right]$. We claim that for any $x \in\{-1,1\}^{n}$, it holds that $\operatorname{Var}_{x_{i}}[\ell(x)] \leq w_{i}^{2}$, which yields the lemma. To show this claim we write $\ell(x)=\left|w_{i} x_{i}+c_{i}\right|$, where $c_{i}=\sum_{j \neq i} w_{j} \cdot x_{j}$ does not depend on $x_{i}$.

Since $\ell^{2}(x)=c_{i}^{2}+w_{i}^{2}+2 c_{i} w_{i} x_{i}$, it follows that $\mathbf{E}_{x_{i}}\left[\ell^{2}(x)\right]=c_{i}^{2}+w_{i}^{2}$, and clearly $\mathbf{E}_{x_{i}}[\ell(x)]=$ $(1 / 2) \cdot\left(\left|w_{i}-c_{i}\right|+\left|w_{i}+c_{i}\right|\right)$. We consider two cases based on the relative magnitudes of $c_{i}$ and $w_{i}$.

If $\left|c_{i}\right| \leq\left|w_{i}\right|$, we have $\mathbf{E}_{x_{i}}[\ell(x)]=(1 / 2) \cdot\left(\operatorname{sign}\left(w_{i}\right)\left(w_{i}-c_{i}\right)+\operatorname{sign}\left(w_{i}\right)\left(w_{i}+c_{i}\right)\right)=\left|w_{i}\right|$. Hence, in this case $\operatorname{Var}_{x_{i}}[\ell(x)]=c_{i}^{2} \leq w_{i}^{2}$. If on the other hand $\left|c_{i}\right|>\left|w_{i}\right|$, then we have $\mathbf{E}_{x_{i}}[\ell(x)]=(1 / 2)$. $\left(\operatorname{sign}\left(c_{i}\right)\left(c_{i}-w_{i}\right)+\operatorname{sign}\left(c_{i}\right)\left(c_{i}+w_{i}\right)\right)=\left|c_{i}\right|$, so again $\operatorname{Var}_{x_{i}}[\ell(x)]=w_{i}^{2}$ as desired.

The bound $\mathbf{K}(w) \geq 1 / \sqrt{2}$ follows from the above two claims using the fact that $\ell$ is even and that $\sum_{i} w_{i}^{2}=1$.

\subsection{Proof of Theorem 22}

Let $w \in \mathbb{R}^{n}$ be a proper unit vector and denote $\tau=\left\|w-w^{*}\right\|_{2}$. To prove Theorem 22, one would intuitively want to obtain a robust version of the simple Fourier-analytic proof of Theorem 21 from the previous subsection. Recall that the latter proof boils down to the following:

$$
\operatorname{Var}[\ell] \leq(1 / 2) \cdot \operatorname{Inf}(\ell)=(1 / 2) \cdot \sum_{i=1}^{n} \operatorname{Inf}_{i}(\ell) \leq(1 / 2) \cdot \sum_{i=1}^{n} w_{i}^{2}=1 / 2
$$

where the first inequality is Fact 23 and the second is Lemma 24. While it is clear that both inequalities can be individually tight, one could hope to show that both inequalities cannot be tight simultaneously. It turns out that this intuition is not quite true, however it holds if one imposes some additional conditions on the weight vector $w$. The remaining cases for $w$ that do not satisfy these conditions can be handled by elementary arguments.

We first note that without loss of generality we may assume that $w_{1}=\max _{i} w_{i}>0.3$, for otherwise Theorem 22 follows directly from the following result of König et al:

Theorem 25 ([KSTJ99]). For a proper unit vector $w \in \mathbb{R}^{n}$, we have $\mathbf{K}(w) \geq \sqrt{2 / \pi}-(1-\sqrt{2 / \pi}) w_{1}$.

Indeed, if $w_{1} \leq 0.3$, the above theorem gives that

$$
\mathbf{K}(w) \geq 1.3 \sqrt{2 / \pi}-0.3>0.737>1 / \sqrt{2}+3 / 100 \geq 1 / \sqrt{2}+(1 / 50) \tau,
$$

where the last inequality follows from the fact that $\tau \leq \sqrt{2}$ (as both $w$ and $w^{*}$ are unit vectors). Hence, we will henceforth assume that $w_{1}>0.3$. (We note that there is nothing special about the number 0.3 ; by adjusting various constants elsewhere in the argument, our proof can be made to work with 0.3 replaced by any (smaller) absolute positive constant. As a result, we could have avoided using Theorem 25 and used quantitatively weaker versions of the theorem which can be shown to follow easily from the Berry-Esséen theorem. However, for convenience we have used Theorem 25 and the number 0.3 in what follows.)

The preceding discussion leads us to the following definition: 
Definition 26 (canonical vector). We say that a proper unit vector $w \in \mathbb{R}^{n}$ is canonical if it satisfies the following conditions:

(a) $w_{1} \in[0.3,1 / \sqrt{2}+1 / 100]$;

(b) $\tau=\left\|w-w^{*}\right\|_{2} \geq 2 / 5$;

The following lemma establishes Theorem 22 for non-canonical vectors:

Lemma 27. Let $w$ be a proper non-canonical vector. Then $\mathbf{K}(w) \geq 1 / \sqrt{2}+(1 / 1000) \tau$, where $\tau=$ $\left\|w-w^{*}\right\|_{2}$.

The proof of Lemma 27 is elementary, using only basic facts about symmetric random variables, but sufficiently long that we give it in Section 3.3 For canonical vectors we show:

Theorem 28. There exist universal constants $c_{1}, c_{2}>0$ such that: Let $w \in \mathbb{R}^{n}$ be canonical. Consider the mapping $\ell(x)=|w \cdot x|$. Then at least one of the following statements is true :

(1) $\operatorname{Inf}_{1}(\ell) \leq w_{1}^{2}-c_{1}$;

(2) $\mathbf{W}^{>2}[\ell] \geq c_{2}$.

This proof is more involved, using Fourier analysis and critical index arguments. We defer it to Section 3.4 and proceed now to show that for canonical vectors, Theorem 22 follows from Theorem 28 . To see this we argue as follows: Let $w \in \mathbb{R}^{n}$ be canonical. We will show that there exists a universal constant $c>0$ such that $\mathbf{K}(w) \geq 1 / \sqrt{2}+c$; as mentioned above, since $\tau<\sqrt{2}$, this is sufficient for our purposes. Now recall that

$$
\mathbf{K}(w)=\mathbf{E}_{x}[\ell(x)]=\widehat{\ell}(0)=\sqrt{1-\operatorname{Var}[\ell]} .
$$

In both cases, we will show that there exists a constant $c^{\prime}>0$ such that

$$
\operatorname{Var}[\ell] \leq 1 / 2-c^{\prime}
$$

From this (4) gives $\mathbf{K}(w) \geq \sqrt{1 / 2+c^{\prime}}=1 / \sqrt{2}+c^{\prime \prime}$ where $c^{\prime \prime}>0$ is a universal constant, so to establish Theorem 22 it suffices to establish (5).

Suppose first that statement (1) of Theorem 28 holds. In this case we exploit the fact that Lemma 24 is not tight. We can write

$$
\operatorname{Var}[\ell] \leq(1 / 2) \cdot \operatorname{Inf}(f) \leq(1 / 2) \cdot\left(w_{1}^{2}-c_{1}+\sum_{i=2}^{n} w_{i}^{2}\right) \leq(1 / 2)-c_{1} / 2,
$$

giving (5). Now suppose that statement (2) of Theorem 28 holds, i.e. at least a $c_{2}$ fraction of the total Fourier mass of $\ell$ lies above level 2. Since $\ell$ is even, this is equivalent to the statement $\mathbf{W}^{\geq 4}[\ell] \geq c_{2}$. In this case, we prove a better upper bound on the variance because Fact 23 is not tight. In particular, we have

$$
\operatorname{Inf}(\ell) \geq 2 \mathbf{W}^{2}[\ell]+4 \mathbf{W}^{\geq 4}[\ell]=2\left(\operatorname{Var}[\ell]-\mathbf{W}^{\geq 4}[\ell]\right)+4 \mathbf{W}^{\geq 4}[\ell]=2 \operatorname{Var}[\ell]+2 \mathbf{W}^{\geq 4}[\ell]
$$

which yields $\operatorname{Var}[\ell] \leq(1 / 2) \operatorname{Inf}(\ell)-\mathbf{W}^{\geq 4}[\ell] \leq(1 / 2)-c_{2}$, again giving (5) as desired. 


\subsection{Proof of Lemma 27}

We will need the following important claim for the proof of Lemma 27

Claim 29. Let $X$ be a symmetric discrete random variable supported on $\mathbb{R}$, i.e. $\operatorname{Pr}[X=x]=\operatorname{Pr}[X=-x]$ for all $x \in \mathbb{R}$. Then for all $c \in \mathbb{R}$ we have

$$
\max \{\mathbf{E}[|X|],|c|\} \leq \mathbf{E}[|X+c|] .
$$

Proof. Since $X$ is symmetric, $c+X$ and $c-X$ have the same distribution. As a result, we have $\mathbf{E}[|X+c|]=$ $(1 / 2) \cdot(\mathbf{E}[|c+X|]+\mathbf{E}[|c-X|])$. Further,

$$
\begin{aligned}
(1 / 2) \cdot(\mathbf{E}[|c+X|]+\mathbf{E}[|c-X|]) & =\mathbf{E}[(1 / 2) \cdot(|c+X|+|c-X|)] \\
& =\mathbf{E}[\max \{|X|,|c|\}] \geq \max \{\mathbf{E}[|X|], c\}
\end{aligned}
$$

which finishes the proof.

Proof of Lemma 27, If $w$ is a non-canonical vector, then there are exactly two possibilities :

Case 1: $w_{1} \notin[0.3,1 / \sqrt{2}+1 / 100]$. In case $w_{1} \leq 0.3$, then the calculation following Theorem 25$]$ already gives us that $\mathbf{K}(w) \geq 1 / \sqrt{2}+(1 / 50) \tau$. The other possibility is that $w_{1} \geq 1 / \sqrt{2}+1 / 100$. In this case,

$$
\begin{aligned}
\mathbf{K}(w)=\mathbf{E}\left[\left|\sum_{i=1}^{n} w_{i} x_{i}\right|\right] & =(1 / 2) \cdot \mathbf{E}\left[\left|w_{1}+\sum_{i=2}^{n} w_{i} x_{i}\right|\right]+(1 / 2) \cdot \mathbf{E}\left[\left|-w_{1}+\sum_{i=2}^{n} w_{i} x_{i}\right|\right] \\
& \geq \frac{\left|w_{1}\right|+\left|w_{1}\right|}{2}=\left|w_{1}\right|
\end{aligned}
$$

where the inequality is an application of Claim 29 As $\left|w_{1}\right| \geq 1 / \sqrt{2}+1 / 100$, we get that

$$
\mathbf{K}(w) \geq 1 / \sqrt{2}+1 / 100 \geq 1 / \sqrt{2}+1 / 200 \cdot \tau
$$

(using that $\tau \leq \sqrt{2}$ ).

Case 2: $\tau \leq 2 / 5$. Of course, here we can also assume that $w_{1} \in[0.3,1 / \sqrt{2}+1 / 100]$ (since otherwise, Case 1 proves the claim). We let $w_{1}=1 / \sqrt{2}-a$ and $w_{2}=1 / \sqrt{2}-b$ and $\sum_{i>2} w_{i}^{2}=c^{2}$. By definition, we have that $a \leq b$ and $b \geq 0$. Also,

$$
\tau^{2}=\left\|w-w^{*}\right\|_{2}^{2}=a^{2}+b^{2}+c^{2} .
$$

Moreover, since $w$ is a unit vector, we have that

$$
a^{2}+b^{2}+c^{2}=\sqrt{2}(a+b) .
$$

Expanding the expression for $\mathbf{K}(w)$ on $x_{1}, x_{2}$ and recalling that $x^{(2)}=\left(x_{3}, \ldots, x_{n}\right)$, we get

$$
\begin{aligned}
& \mathbf{K}(w)=\frac{1}{2} \cdot\left(\mathbf{E}_{x^{(2)} \in\{-1,1\}^{n-2}}\left[\left|\sqrt{2}-(a+b)+w^{(2)} \cdot x^{(2)}\right|\right]\right. \\
&\left.+\mathbf{E}_{x^{(2)} \in\{-1,1\}^{n-2}}\left[\left|(a-b)+w^{(2)} \cdot x^{(2)}\right|\right]\right) \\
& \geq \frac{1}{2} \cdot\left(\max \left\{|\sqrt{2}-(a+b)|, \mathbf{E}\left[\left|w^{(2)} \cdot x^{(2)}\right|\right]\right\}+\max \left\{|a-b|, \mathbf{E}\left[\left|w^{(2)} \cdot x^{(2)}\right|\right]\right\}\right) \\
& \geq \frac{1}{2} \cdot\left(\max \left\{|\sqrt{2}-(a+b)|, \frac{c}{\sqrt{2}}\right\}+\max \left\{|a-b|, \frac{c}{\sqrt{2}}\right\}\right)
\end{aligned}
$$


where the first inequality follows from Claim 29 and the second inequality uses the fact $\mathbf{E}\left[\left|w^{(2)} \cdot x^{(2)}\right|\right] \geq$ $c / \sqrt{2}$ (as follows from Theorem 7). We consider two further sub-cases :

Case 2(a): Let $c^{2} \geq \tau^{2} / 20$. Then, we can bound the right hand-side from below as follows:

$$
\begin{aligned}
& \frac{1}{2} \cdot\left(\max \left\{|\sqrt{2}-(a+b)|, \frac{c}{\sqrt{2}}\right\}+\max \left\{|a-b|, \frac{c}{\sqrt{2}}\right\}\right) \geq \frac{1}{2} \cdot\left(|\sqrt{2}-(a+b)|+\frac{c}{\sqrt{2}}\right) \\
\geq & \frac{1}{2}\left(\left|\sqrt{2}-\frac{\tau^{2}}{\sqrt{2}}\right|\right)+\frac{\tau}{4 \sqrt{10}}=\frac{1}{\sqrt{2}}-\frac{\tau^{2}}{2 \sqrt{2}}+\frac{\tau}{\sqrt{40}}
\end{aligned}
$$

where the second inequality uses $(7)$. As long as $\tau \leq 2 / 5$, it is easy to check that

$$
\frac{\tau}{\sqrt{40}}-\frac{\tau^{2}}{2 \sqrt{2}} \geq \frac{\tau}{1000}
$$

which proves the assertion in this case.

Case 2(b): Let $c^{2}<\tau^{2} / 20$. In this case, we will prove a lower bound on $|a-b|$. Using $c^{2}<\tau^{2} / 20$ and (6), we have $a^{2}+b^{2}>\left(19 \tau^{2}\right) / 20$. Also, using (7), we have $a+b=\tau^{2} / \sqrt{2}$. We now have

$$
(a-b)^{2}=2\left(a^{2}+b^{2}\right)-(a+b)^{2} \geq 2 \cdot \frac{19}{20} \cdot \tau^{2}-\frac{\tau^{4}}{2} \geq \frac{19}{10} \tau^{2}-\frac{\tau^{4}}{2} \geq \tau^{2}
$$

The last inequality uses $\tau \leq 2 / 5$. Now, as in Case 2(a), we have

$$
\begin{aligned}
& \frac{1}{2} \cdot\left(\max \left\{|\sqrt{2}-(a+b)|, \frac{c}{\sqrt{2}}\right\}+\max \left\{|a-b|, \frac{c}{\sqrt{2}}\right\}\right) \geq \frac{1}{2} \cdot(|\sqrt{2}-(a+b)|+|a-b|) \\
\geq & \frac{1}{2}\left(\left|\sqrt{2}-\frac{\tau^{2}}{\sqrt{2}}\right|\right)+\frac{\tau}{2}=\frac{1}{\sqrt{2}}-\frac{\tau^{2}}{2 \sqrt{2}}+\frac{\tau}{2} \geq \frac{1}{2}+\frac{\tau}{1000}
\end{aligned}
$$

Again, the last inequality uses that $\tau \leq 2 / 5$. This finishes the proof of Lemma 27

\subsection{Proof of Theorem 28}

We will prove that if $w \in \mathbb{R}^{n}$ is a canonical vector such that $\operatorname{Inf}_{1}(\ell) \geq w_{1}^{2}-c_{1}$, then $\mathbf{W}^{>2}[\ell] \geq c_{2}$. For the sake of intuition, we start by providing a proof sketch for the special case that $c_{1}=0$. At a high-level, the actual proof will be a robust version of this sketch using the notion of the critical index to make the simple arguments for the " $c_{1}=0$ case" robust. For this case, it suffices to prove the following implication:

If $\operatorname{Inf}_{1}(\ell)=w_{1}^{2}$, then at least a constant fraction of the Fourier weight of $\ell$ lies above level 2.

Indeed, we have the following claims:

(1) Let $w$ be canonical and $\operatorname{Inf}_{1}(\ell)=w_{1}^{2}$. Then $w$ equals $\left(w_{1}, \ldots, w_{1}, 0, \ldots, 0\right)$ where there are $k$ repetitions of $w_{1}$ and $k$ is even. We call such a $w$ "good".

(2) Let $w$ be a good vector. Then $\ell$ has $\Theta(1)$ Fourier weight above level 2.

We can prove (1) as follows. Suppose that $\operatorname{Inf}_{1}(\ell)=w_{1}^{2}$. Then, as implied by the proof of Lemma 24 every outcome $\rho^{(2)}$ of $\left(x_{2}, \ldots, x_{n}\right)$ has $\left|w^{(2)} \cdot \rho^{(2)}\right| \geq w_{1}$. Suppose, for the sake of contradiction, that some coordinate $w_{j}$ is neither equal to $w_{1}$ nor to 0 . Let $w_{k}(k \geq 2)$ be the first such value. By having $\rho_{2}, \ldots, \rho_{k-1}$ alternate between +1 and -1 we can ensure that there is an assignment of $\rho_{2}, \ldots, \rho_{k-1}$ such that $w_{2} \rho_{2}+\cdots+w_{k-1} \rho_{k-1}$ is either 0 (if $k$ is even) or $w_{1}$ (if $k$ is odd). In the former case, by choosing the remaining $\rho$ bits appropriately we get that there exists an assignment $\rho$ such that $\left|w^{(2)} \cdot \rho^{(2)}\right| \leq w_{k}<w_{1}$, 
where the inequality uses the fact that the $w_{i}$ 's are non-increasing and our assumption that $w_{k} \neq w_{1}$. In the latter case, if $w_{k}$ is the last nonzero entry, for an appropriate $\rho$, we can get $\left|w^{(2)} \cdot \rho^{(2)}\right|=w_{1}-w_{k}<w_{1}$. Otherwise, if there are other nonzero entries beyond $w_{k}$ we can similarly get $\left|w^{(2)} \cdot \rho^{(2)}\right|<w_{k}$. So we have argued that if there is any $w_{k} \notin\left\{0, w_{1}\right\}$ then it cannot be the case that $\operatorname{Inf}_{1}(\ell)=w_{1}^{2}$, so $w$ must be of the form ( $k$ copies of $w_{1}$ followed by 0 's). If $k$ is odd, then clearly there exists a $\rho$ such that $\left|w^{(2)} \cdot \rho^{(2)}\right|=0$. So, it must be the case that $k$ is even. This proves (1). Given (1) in hand, we may conclude (2) using the following lemma (Lemma 30) and the observation (recalling that $w$ is canonical) that since $w_{1} \geq 0.3$ we must have $k \leq 12$ :

Lemma 30. Let $\ell_{k}(x)=\left|\frac{\left(x_{1}+\ldots+x_{k}\right)}{\sqrt{k}}\right|$. For $k \geq 4$ and even, $\mathbf{W}^{\geq 4}[\ell] \geq \frac{2^{-2 k}}{k}$.

Proof. We start by observing that because $\ell_{k}(x)$ only takes values which are integral multiples of $k^{-1 / 2}$, it must be the case that for any character $\chi_{S}$, the value $\widehat{\ell_{k}}(S)=\mathbf{E}\left[\chi_{S}(x) \cdot \ell_{k}(x)\right]$ is a multiple of $2^{-k} \cdot k^{-1 / 2}$. Hence, any non-zero Fourier coefficient of $\ell_{k}$ is at least $2^{-k} \cdot k^{-1 / 2}$ in magnitude. Thus, if $\mathbf{W}^{\geq 4}[\ell] \neq 0$, then $\mathbf{W}^{\geq 4}[\ell] \geq k^{-1} 2^{-2 k}$. Thus, to prove the lemma, we need to show that $\mathbf{W}^{\geq 4}[\ell] \neq 0$.

Next, we observe that $\ell_{k}(x)$ is an even function and hence any Fourier coefficient $\hat{f}(S)=0$ if $|S|$ is odd. Thus, towards a contradiction, if we assume that $\mathbf{W}^{\geq 4}[\ell]=0$, then the Fourier expansion of $\ell_{k}(x)$ must consist solely of a constant term and degree 2 terms. As the function $\ell_{k}(x)$ is symmetric, we may let the coefficient of any quadratic term be $\alpha$ and the constant term be $\beta$, and we have

$$
\begin{aligned}
\ell_{k}(x) & =\beta+\sum_{i<j} \alpha \cdot x_{i} x_{j}=\beta+\alpha \cdot\left(\sum_{i<j} x_{i} x_{j}\right)=\beta+\frac{\alpha \cdot\left(\left(\sum_{i=1}^{k} x_{i}\right)^{2}-\sum_{i=1}^{k} x_{i}^{2}\right)}{2} \\
& =\beta+\frac{\alpha \cdot\left(\left(\sum_{i=1}^{k} x_{i}\right)^{2}-k\right)}{2}=\frac{\alpha}{2} \cdot\left(\sum_{i=1}^{k} x_{i}\right)^{2}+\beta-\frac{\alpha k}{2}=\gamma_{1}\left(\sum_{i=1}^{k} x_{i}\right)^{2}+\gamma_{2}
\end{aligned}
$$

where $\gamma_{1}=\alpha / 2$ and $\gamma_{2}=\beta-\frac{\alpha k}{2}$. Note that since $k$ is even, there exist assignments $x \in\{-1,1\}^{k}$ that cause $\sum_{i=1}^{k} x_{i}$ to take any even value in $[-k, k]$; in particular, since $k \geq 4$, the sum $\sum_{i=1}^{k} x_{i}$ may take any of the values $0,2,4$.

Now, if $\sum_{i=1}^{k} x_{i}=0$, then $\ell_{k}(x)=0$. Hence we infer that $\gamma_{2}=0$. If $\sum_{i=1}^{k} x_{i}=2$ then $\ell_{k}(x)=2 / \sqrt{k}$, and if $\sum_{i=1}^{k} x_{i}=4$ then $\ell_{k}(x)=4 / \sqrt{k}$. Clearly, there is no $\gamma_{1}$ satisfying both $\gamma_{1} \cdot 2^{2}=2 / \sqrt{k}$ and $\gamma_{1} \cdot 4^{2}=4 / \sqrt{k}$. This gives a contradiction. Hence $\mathbf{W} \geq 4[\ell] \neq 0$ and the lemma is proved.

We can now proceed with the formal proof of Theorem 28 We will need several facts and intermediate lemmas. The first few facts show some easy concentration properties for weighted linear combinations of random signs under certain conditions on the weights.

Claim 31. Fix $\alpha>0$. Let $w_{1}, \ldots, w_{n} \in \mathbb{R}$ satisfy $\left|w_{i}\right| \leq \alpha$ for all $i$. Then there exists $x^{*} \in\{-1,1\}^{n}$ such that $w \cdot x \in[0, \alpha]$ (and clearly $-x^{*} \in\{-1,1\}^{n}$ has $w \cdot\left(-x^{*}\right) \in[-\alpha, 0]$ ).

Proof. Construct $x^{\prime} \in\{-1,1\}^{n}$ one bit at a time, by choosing $x_{i+1}^{\prime}$ so that $\operatorname{sign}\left(w_{i+1} x_{i+1}^{\prime}\right)=-\operatorname{sign}\left(w_{1} x_{1}^{\prime}+\right.$ $\left.\cdots+w_{i} x_{i}^{\prime}\right)$. The resulting vector $x^{\prime}$ satisfies $\left|w \cdot x^{\prime}\right| \leq \alpha$.

As a special case of this we get:

Claim 32. Fix $0<\eta \leq \alpha$. Let $w_{j} \in \mathbb{R}^{+}, j \in[2 k+1]$, satisfy $w_{j} \in[\alpha-\eta, \alpha]$. Then, there exists $x^{*}=\left(x_{1}^{*}, \ldots, x_{2 k+1}^{*}\right) \in\{-1,1\}^{2 k+1}$ such that: $\sum_{j=1}^{2 k+1} w_{j} x_{j}^{*} \in[0, \alpha]$.

The following claim is only slightly less immediate: 
Claim 33. Fix $0<\eta \leq \alpha$. Let $w_{j} \in \mathbb{R}^{+}, j \in[2 k]$, satisfy $w_{j} \in[\alpha-\eta, \alpha]$. Then, there exists $x^{*}=$ $\left(x_{1}^{*}, \ldots, x_{2 k}^{*}\right) \in\{-1,1\}^{2 k}$ such that: $\sum_{j=1}^{2 k} w_{j} x_{j}^{*} \in[0, \eta]$.

Proof. The vector $u \in \mathbb{R}^{k}$ defined by $u_{j}=w_{2 j}-w_{2 j-1}$ has $\left|u_{j}\right| \leq \eta$ for all $j \in[k]$. It is clear that the set of values $\{w \cdot x\}_{x \in\{-1,1\}^{2 k}}$ is contained in $\{u \cdot x\}_{x \in\{-1,1\}^{k}}$. The claim follows by applying Claim 31 to $u$.

We will also need the following corollary of the Berry-Esséen theorem (more precisely, it follows from Fact 17 together with the fact that the pdf of a standard Gaussian has value at least 0.2 everywhere on $[-1,1])$ :

Fact 34. Fix $0<\tau<1 / 15$. Let $w \in \mathbb{R}^{n}$ be $\tau$-regular with $\|w\|_{2} \leq 1$. Then, $\operatorname{Pr}_{x}[0 \leq w \cdot x \leq 15 \tau] \geq \tau$ and $\mathbf{P r}_{x}[-15 \tau \leq w \cdot x \leq 0] \geq \tau$.

We are now ready to prove the following lemma which establishes a concentration statement for linear forms with a given maximum coefficient:

Lemma 35. Let $w \in \mathbb{R}^{n}$ be proper with $\|w\|_{2} \leq 1$ and let $\delta \stackrel{\text { def }}{=} w_{1}>0$. There exists $\kappa=\kappa(\delta)$ such that $\operatorname{Pr}_{x}[0 \leq w \cdot x \leq \delta] \geq \kappa$.

Proof. We choose a sufficiently small $\tau>0$, where $\tau=\tau(\delta) \ll \delta$, and consider the $\tau$-critical index $K=c(w, \tau)$ of $w$. Fix $K_{0}=\Theta\left(1 / \tau^{2}\right) \cdot \log \left(1 / \delta^{2}\right)$ and consider the following two cases:

[Case 1: $K \leq K_{0}$.] In this case, we partition $[n]$ into the head $H=[K-1]$ and the tail $T=[n] \backslash H$. Then, an application of Claims 33 and 32 for $\eta=\alpha=\delta$ gives us that

$$
\operatorname{Pr}_{x_{H}}\left[w_{H} \cdot x_{H} \in[0, \delta]\right] \geq 2^{-K} \geq 2^{-K_{0}}
$$

An application of Fact 34 for the $\tau$-regular tail gives that

$$
\mathbf{P r}_{x_{T}}\left[w_{T} \cdot x_{T} \in[-15 \tau, 0]\right] \geq \tau
$$

and combining the above inequalities using independence yields

$$
\operatorname{Pr}_{x}[w \cdot x \in[-15 \tau, \delta]] \geq 2^{-K_{0}} \cdot \tau
$$

Now note that for any choice of $\tau \leq \delta / 15$, the above clearly implies

$$
\operatorname{Pr}_{x}[w \cdot x \in[-\delta, \delta]] \geq 2^{-K_{0}} \cdot \tau
$$

and by symmetry we conclude that

$$
\operatorname{Pr}_{x}[w \cdot x \in[0, \delta]] \geq 2^{-K_{0}-1} \cdot \tau
$$

yielding the lemma for $\kappa_{1}=2^{-K_{0}-1} \cdot \tau$.

[Case 2: $L>K_{0}$.] In this case, we partition $[n]$ into $H=\left[K_{0}-1\right]$ and the tail $T=[n] \backslash H$. We similarly have that

$$
\operatorname{Pr}_{x_{H}}\left[w_{H} \cdot x_{H} \in[0, \delta]\right] \geq 2^{-K_{0}} .
$$

Now recall that the tail weight decreases geometrically up to the critical index; in particular, Fact 18 gives that $\left\|w_{T}\right\|_{2} \leq \delta^{2}$. Then, for a sufficiently small $\delta$, the Hoeffding bound gives

$$
\operatorname{Pr}_{x_{T}}\left[w_{T} \cdot x_{T} \in[-\delta, 0]\right] \geq 1 / 4 \text {. }
$$


Combining these inequalities we thus get that

$$
\operatorname{Pr}_{x}[w \cdot x \in[-\delta, \delta]] \geq 2^{-K_{0}-2} .
$$

By symmetry, we get the desired inequality for $\kappa_{2}=2^{-K_{0}-3}$.

The proof follows by selecting $\kappa=\min \left\{\kappa_{1}, \kappa_{2}\right\}=\kappa_{1}$ for any choice of $\tau \leq \delta / 15$.

Note the difference between the conditions of Corollary 36, stated below, and Lemma 35 stated above: while Lemma 35 requires that $\delta=w_{1}$, Corollary 36 holds for any $\delta>0$.

Corollary 36. For any $\delta>0$, there is a value $\kappa=\kappa(\delta)>0$ such that for any $w \in \mathbb{R}^{n}$ with $\|w\|_{2} \leq 1$ and $\|w\|_{\infty} \leq \delta, \mathbf{P r}_{x}[0 \leq w \cdot x \leq \delta] \geq \kappa$ and $\mathbf{P r}_{x}[-\delta \leq w \cdot x \leq 0] \geq \kappa$.

Proof. We start by considering the case when $\|w\|_{2} \leq \delta / 100$. In this case, by Theorem 15, we certainly get that $\operatorname{Pr}_{x}[|w \cdot x| \leq \delta] \geq 99 / 100$. Hence, by symmetry, $\operatorname{Pr}_{x}[-\delta \leq w \cdot x \leq 0] \geq 99 / 200$ and $\operatorname{Pr}_{x}[0 \leq w \cdot x \leq \delta] \geq 99 / 200$.

Next, we consider the case when $\|w\|_{2}>\delta / 1500$. In this case, if $w_{1}>\delta^{2} / 1500$, then we apply Lemma 35, to get that $\operatorname{Pr}_{x}\left[0 \leq w \cdot x \leq \delta^{2} / 1500\right] \geq \kappa_{1}$ and (by symmetry) $\operatorname{Pr}_{x}\left[-\delta^{2} / 1500 \leq w \cdot x \leq 0\right] \geq$ $\kappa_{1}$ where $\kappa_{1}$ is a positive constant dependent only on $\delta$.

The only remaining case is when $w_{1} \leq \delta^{2} / 1500$. In this case, the vector $w$ is $\delta / 15$-regular. Now, we can apply Fact 34 to get that $\operatorname{Pr}_{x}[0 \leq w \cdot x \leq \delta] \geq \delta / 15$ and $\operatorname{Pr}_{x}[-\delta \leq w \cdot x \leq 0] \geq \delta / 15$. By taking $\kappa=\min \left\{\delta / 15, \kappa_{1}, 99 / 200\right\}$, the proof is completed.

Using the above corollary, we show the following lemma:

Lemma 37. Let $\alpha, \eta, \xi \in \mathbb{R}^{+}$with $w \in \mathbb{R}^{n}$ be such that $\xi \leq\|w\|_{2} \leq 1, \alpha>2 \eta$, and $\|w\|_{\infty} \leq \alpha-\eta$. Then, there are positive constants $\kappa=\kappa(\alpha, \eta, \xi)$ and $\gamma=\gamma(\alpha, \eta, \xi)$ such that

$$
\operatorname{Pr}_{x}[-2 \alpha+2 \eta \leq w \cdot x \leq-\gamma] \geq \kappa
$$

Proof. We choose a sufficiently small $\zeta>0$ and consider two cases.

[Case 1: $w$ is $\zeta$-regular.] In this case, Theorem 16 gives us (similar to Fact 34) that for $\zeta \leq 1 / 20$, we have

$$
\operatorname{Pr}_{x}[-20 \zeta \cdot\|w\| \leq w \cdot x \leq-\zeta \cdot\|w\|] \geq \zeta
$$

[Case 2: $w$ is not $\zeta$-regular.] We assume without loss of generality that $w_{1}=\|w\|_{\infty}$. In this case, it follows by definition that $w_{1} \geq \zeta \cdot \xi$, hence $w_{1} \in[\zeta \cdot \xi, \alpha-\eta]$. Since $\left|w_{j}\right| \leq \alpha-\eta$ for all $j \geq 2$, Corollary 36 says that $\left(\right.$ recall that $\left.w^{(1)}=\left(w_{2}, \ldots, w_{n}\right)\right)$

$$
\operatorname{Pr}_{x^{(1)}}\left[-\alpha+\eta \leq w^{(1)} \cdot x^{(1)} \leq 0\right] \geq c(\alpha, \eta) .
$$

By independence we thus get

$$
\operatorname{Pr}_{x}[-2 \alpha+2 \eta \leq w \cdot x \leq-\zeta \cdot \xi] \geq c(\alpha, \eta) / 2 .
$$

Combining Case 1 and Case 2 and using $1 \geq\|w\| \geq \xi$, we get

$$
\operatorname{Pr}_{x}[\min \{-2 \alpha+2 \eta,-20 \zeta\} \leq w \cdot x \leq-\zeta \cdot \xi] \geq \min \{c(\alpha, \eta) / 2, \zeta\}
$$

We now choose $\zeta>0$ so that $20 \zeta \leq \alpha-\eta$. Finally, we set $\gamma=\zeta \cdot \xi$ and $\kappa=\min \{c(\alpha, \eta) / 2, \zeta\}$ and get the claimed result. 
The next lemma is a robust version of Lemma 30. It says that if a vector $w$ of length $n$ is very close to having its first $2 k$ entries each being $\alpha$ and its remaining entries all 0 , then $\ell(x)=|w \cdot x|$ must have nonnegligible Fourier mass at levels 4 and above.

Lemma 38. Let $\alpha>0, w \in \mathbb{S}^{n-1}$ and $k \in \mathbb{N}, k>1$. Then there are sufficiently small positive constants $\eta=\eta(k), \tau=\tau(k)$ with the following property : If for every $i \in[2 k]$, we have $w_{i} \in[\alpha-\eta, \alpha]$ and $\sum_{j>2 k}^{n}\left(w_{j}\right)^{2} \leq \tau^{2}$, then the map $\ell: x \mapsto|w \cdot x|$ satisfies $\mathbf{W}^{\geq 4}[\ell] \geq \gamma$ for some $\gamma=\gamma(k)>0$.

Proof. Consider the vector $w^{\prime}=(\underbrace{\alpha, \ldots, \alpha}_{2 k}, 0, \ldots, 0)$ and the map $\ell^{\prime}: x \mapsto\left|w^{\prime} \cdot x\right|$. We have

$$
\ell^{\prime}(x)=\alpha \cdot \sqrt{k} \cdot\left|\frac{x_{1}+\ldots+x_{k}}{\sqrt{k}}\right|
$$

By applying Lemma 30, we get $\mathbf{W}^{\geq 4}\left[\ell^{\prime}\right] \geq \alpha^{2} \cdot 2^{-2 k}$. Note that if $\eta$ and $\tau$ are sufficiently small, then clearly $\alpha \geq \frac{1}{2 \sqrt{k}}$. This implies $\mathbf{W}^{\geq 4}\left[\ell^{\prime}\right] \geq \frac{2^{-2 k}}{4 k}$.

We now observe that

$$
\begin{aligned}
\left|\ell(x)-\ell^{\prime}(x)\right| & =|| \sum_{i=1}^{n} w_{i} \cdot x_{i}|-| \sum_{i=1}^{n} w_{i}^{\prime} \cdot x_{i}|| \\
& \leq|| \sum_{i=1}^{k} w_{i} \cdot x_{i}|-| \sum_{i=1}^{k} w_{i}^{\prime} \cdot x_{i}||+\left|\sum_{j=k+1}^{n} w_{i} x_{i}\right|
\end{aligned}
$$

Let us use $h_{1}(x)=\left|\sum_{i=1}^{k} w_{i} \cdot x_{i}\right|, h_{2}(x)=\left|\sum_{i=1}^{k} w_{i}^{\prime} \cdot x_{i}\right|$ and $h_{3}(x)=\left|\sum_{j=k+1}^{n} w_{i} x_{i}\right|$. Then we may rewrite the above as $\left|\ell(x)-\ell^{\prime}(x)\right| \leq\left|h_{1}(x)-h_{2}(x)\right|+h_{3}(x)$. This implies that $\left|\ell(x)-\ell^{\prime}(x)\right|^{2} \leq$ $2\left(h_{1}(x)-h_{2}(x)\right)^{2}+2\left(h_{3}(x)\right)^{2}$. This in turn yields

$$
\mathbf{E}\left[\left(\ell(x)-\ell^{\prime}(x)\right)^{2}\right] \leq 2 \mathbf{E}\left[\left(h_{1}(x)-h_{2}(x)\right)^{2}\right]+2 \mathbf{E}\left[\left(h_{3}(x)\right)^{2}\right] .
$$

Note that $\mathbf{E}\left[\left(h_{3}(x)\right)^{2}\right]=\sum_{j=k+1}^{n} w_{j}^{2} \leq \tau^{2}$. Next, observe that

$$
\left|h_{1}(x)-h_{2}(x)\right|=|| \sum_{i=1}^{k} w_{i} \cdot x_{i}|-| \sum_{i=1}^{k} w_{i}^{\prime} \cdot x_{i}|| \leq\left|\sum_{i=1}^{k}\left(w_{i}-w_{i}^{\prime}\right) \cdot x_{i}\right|
$$

Hence, we get that $\mathbf{E}\left[\left(h_{1}(x)-h_{2}(x)\right)^{2}\right] \leq \mathbf{E}\left[\left(\sum_{i=1}^{k}\left(w_{i}-w_{i}^{\prime}\right) \cdot x_{i}\right)^{2}\right] \leq \sum_{i=1}^{k} \eta^{2}=k \eta^{2}$.

Combining these bounds, we get that $\mathbf{E}\left[\left(\ell(x)-\ell^{\prime}(x)\right)^{2}\right] \leq 2\left(k \eta^{2}+\tau^{2}\right)$. Hence, we have that

$$
\mathbf{W}^{\geq 4}[\ell] \geq \mathbf{W}^{\geq 4}\left[\ell^{\prime}\right]-\mathbf{E}\left[\left(\ell(x)-\ell^{\prime}(x)\right)^{2}\right] \geq \frac{2^{-2 k}}{4 k}-2\left(k \eta^{2}+\tau^{2}\right) .
$$

We may choose $\eta$ and $\tau$ small enough so that $\frac{2^{-2 k}}{4 k}-2\left(k \eta^{2}+\tau^{2}\right) \geq \frac{2^{-2 k}}{8 k}$, and the proof is finished.

Given the above lemmas, the proof of Theorem 28 proceeds as follows: Let $w_{1}=\alpha$ and $\eta=\eta(\alpha)>0$ be a sufficiently small constant. Let $L$ be the first index such that $w_{L} \leq \alpha-\eta$. Recalling that $w$ is canonical, since $w_{1}>0.3$ and $\|w\|=1$, it is clear that $L \leq 1 / 0.09<12$. We now consider two cases :

[Case I: $L$ is even] Then by Claim 33, there is a choice of $x_{2}, \ldots, x_{L-1}$, such that $\sum_{k=2}^{L-1} w_{k} x_{k} \in[-\eta, 0]$. Using Corollary 36 and noting that $w_{L} \leq \alpha-\eta$, there is some $\kappa=\kappa(\alpha, \eta)$ such that $\operatorname{Pr}_{x^{(L-1)}}[0 \leq$ $\left.w^{(L-1)} x^{(L-1)} \leq \alpha-\eta\right] \geq \kappa$. By independence, we thus get

$$
\operatorname{Pr}_{x}\left[-\eta \leq w^{(1)} \cdot x^{(1)} \leq \alpha-\eta\right] \geq \kappa \cdot 2^{-L} .
$$


Note that (8) implies (by definition) that $\operatorname{Inf}_{1}(\ell) \leq w_{1}^{2}-c_{1}$, for an appropriate constant $c_{1}=c_{1}(\kappa, L, \eta)>$ 0 .

[Case II: $L$ is odd] Let us choose a sufficiently small $\xi>0$. If $\left\|w^{(L-1)}\right\|_{2}>\xi$, then observe that from Claim 33 (applied to the weights $w_{1}, \ldots, w_{L-1}$ ) there is a choice of $x_{2}, \ldots, x_{L-1}$ satisfying $\sum_{k=2}^{L-1} w_{k} x_{k} \in$ $[\alpha-\eta, \alpha]$, i.e.

$$
\operatorname{Pr}\left[\alpha-\eta \leq \sum_{k=2}^{L-1} w_{k} x_{k} \leq \alpha\right] \geq 2^{-L} .
$$

Combining this with Lemma 37 applied to $w^{(L-1)}$, we get that

$$
\operatorname{Pr}_{x^{(1)}}\left[-\alpha+\eta \leq w^{(1)} \cdot x^{(1)} \leq \alpha-\gamma(\alpha, \eta, \xi)\right] \geq 2^{-L} \cdot \kappa .
$$

Exactly as before, (9) implies (by definition) that $\operatorname{Inf}_{1}(\ell) \leq w_{1}^{2}-c_{1}$, for an appropriate constant $c_{1}>0$.

Now consider the only remaining case which is that $\left\|w^{(L-1)}\right\|_{2} \leq \xi$. Recall that $1<L<12$ and $L$ is odd; we first claim that that $L>3$. Indeed, this must be the case because $L=3$ contradicts (for $\xi$ and $\eta$ sufficiently small) the assumption $\tau \geq 2 / 5$ (recall that $w$ is canonical). Now, since $\ell \leq 11$ and $\eta$ and $\xi$ are sufficiently small, by applying Lemma 38), we get that $\ell$ has a constant fraction of its Fourier mass above level 2, completing the proof. This finishes the proof of Theorem 28

\section{Proof of Theorem 3 using Theorem 9}

We first observe that it suffices to prove the theorem for balanced LTFs, i.e. LTFs $f:\{-1,1\}^{n} \rightarrow\{-1,1\}$ with $\widehat{f}(\emptyset)=\mathbf{E}[f]=0$. (Note that any balanced LTF can be represented with a threshold of 0 , i.e. $f(x)=$ $\operatorname{sign}(w \cdot x)$ for some $w \in \mathbb{R}^{n}$.)

Fact 39. Let $f:\{-1,1\}^{n} \rightarrow\{-1,1\}$ be an $n$-variable LTF. Then there is a balanced $(n+1)$-variable LTF $g:\{-1,1\}^{n+1} \rightarrow\{-1,1\}$ such that $\mathbf{W} \leq 1[f]=\mathbf{W}^{\leq 1}[g]$.

Proof. Let $f(x)=\operatorname{sign}\left(w_{0}+\sum_{i=1}^{n} w_{i} x_{i}\right)$ and note that we may assume that $w_{0} \neq w \cdot x$ for all $x \in$ $\{-1,1\}^{n}$. Consider the $(n+1)$-variable balanced LTF $g:(x, y) \rightarrow\{-1,1\}$, where $y \in\{-1,1\}$, defined by $g(x, y)=\operatorname{sign}\left(w_{0} y+\sum_{i=1}^{n} w_{i} x_{i}\right)$. Then it is easy to see that $\widehat{g}(y)=\mathbf{E}[f]$ and $\widehat{g}(i)=\widehat{f}(i)$ for all $i \in[n]$. Therefore, $\mathbf{W}^{\leq 1}[f]=\mathbf{W}^{1}[g]=\mathbf{W}^{\leq 1}[g]$.

Let $f=\operatorname{sign}(w \cdot x)$ be an LTF. We may assume that $w$ is a proper unit vector, i.e. that $\|w\|_{2}=1$ and $w_{i} \geq w_{i+1}>0$ for $i \in[n-1]$. We can also assume that $w \cdot x \neq 0$ for all $x \in\{-1,1\}^{n}$. We distinguish two cases: If $w$ is "far" from $w^{*}$ (i.e. the worst-case vector for the Khintchine inequality), the desired statement follows immediately from our robust inequality (Theorem 9). For the complementary case, we use a separate argument that exploits the structure of $w$. More formally, we have the following two cases:

Let $\tau>0$ be a sufficiently small universal constant, to be specified.

[Case I: $\left\|w-w^{*}\right\|_{2} \geq \tau$ ]. In this case, Proposition 8 and Theorem 9 give us

$$
\mathbf{W}^{1}[f] \geq(\mathbf{K}(w))^{2} \geq(1 / \sqrt{2}+c \tau)^{2} \geq 1 / 2+\sqrt{2} c \tau
$$

which completes the proof of Theorem 3 for Case I.

[Case II: $\left\|w-w^{*}\right\|_{2} \leq \tau$ ]. In this case the idea is to consider the restrictions of $f$ obtained by fixing the variables $x_{1}, x_{2}$ and argue based on their bias. Recall that for a vector $y=\left(y_{1}, \ldots, y_{n}\right) \in \mathbb{R}^{n}$ and $i \in[n]$ we denote $y^{(i)}=\left(y_{i+1}, \ldots, y_{n}\right)$. We consider the restrictions $f_{i j}:\{-1,1\}^{n-2} \rightarrow\{-1,1\}$ defined by

$$
f_{i j}(y)=\operatorname{sign}\left(w_{1} \cdot(-1)^{i}+w_{2} \cdot(-1)^{j}+w^{(2)} \cdot y\right) .
$$

We fix $\lambda=3 / 4$ and consider the following two subcases: 
(a) $\left(\mathbf{E}_{y}\left[f_{01}(y)\right] \leq \lambda\right)$ In this case the function $f_{01}$ is not very positively biased; we show that the CauchySchwarz inequality is not tight. In particular, the degree- 1 Fourier vector $(\widehat{f}(i))_{i=1, \ldots, n}$ of $f(x)=$ $\operatorname{sign}(w \cdot x)$ and the corresponding weight-vector $w$ form an angle bounded away from zero:

Lemma 40. There are universal constants $\tau, \kappa=\kappa(\tau)>0$ such that the following holds: Let $w \in \mathbb{R}^{n}$ be any proper unit vector such that $\left\|w-w^{*}\right\|_{2} \leq \tau$ and $\mathbf{E}_{y}\left[f_{01}(y)\right] \leq \lambda$ where $f(x)=\operatorname{sign}(w \cdot x)$. Then we have

$$
\mathbf{W}^{1}[f] \geq(1+\kappa) \cdot(\mathbf{K}(w))^{2}
$$

Proof. Note that since $w_{1} \geq w_{2}$ the function $f_{01}(y)$ is an LTF of the form $\operatorname{sign}\left(w^{(2)} \cdot y^{(2)}+\theta\right)$ with $\theta \geq 0$, and hence $\mathbf{E}\left[f_{01}\right] \geq 0$. To deal with this case we recall the following simple fact:

Fact 41 (Lemma 2.4 in [OS11]). Let $f:\{-1,1\}^{n} \rightarrow\{-1,1\}$ be an LTF with $1-|\mathbf{E}[f]|=p$. Then $\mathbf{W}^{1}[f] \geq p^{2} / 2$.

An application of Fact 41 for $f_{01}$ gives

$$
\mathbf{W}^{1}\left[f_{01}\right] \geq 1 /(32) .
$$

Note that by symmetry we also have that $\mathbf{E}_{y}\left[f_{10}(y)\right] \geq-\lambda$ and therefore

$$
\mathbf{W}^{1}\left[f_{10}\right] \geq 1 /(32) \text {. }
$$

Fix $k \in\{3, \ldots, n\}$. We have that

$$
\begin{aligned}
\widehat{f}(k) & =\operatorname{Inf}_{k}(f) \\
& =(1 / 4) \cdot \sum_{i, j \in\{0,1\}} \operatorname{Inf}_{k-2}\left(f_{i j}\right) \geq(1 / 4) \cdot\left(\widehat{f_{01}}(k-2)+\widehat{f_{10}}(k-2)\right) .
\end{aligned}
$$

Since the sign of $\widehat{f_{01}}(k-2)$ agrees with the sign of $\widehat{f_{10}}(k-2)$ for all $k \in\{3, \ldots, n\}$, we get that

$$
\sum_{k=3}^{n} \widehat{f}(k)^{2} \geq(1 / 16) \cdot\left(\mathbf{W}^{1}\left[f_{01}\right]+\mathbf{W}^{1}\left[f_{10}\right]\right) \geq 1 /(256) .
$$

Recall that by assumption of the lemma it holds $\left\|w^{(2)}\right\|_{2}=\sqrt{\sum_{i=3}^{n} w_{i}^{2}} \leq \tau$ and Parseval's identity implies that $\sum_{i=1}^{n} \widehat{f}^{2}(i)^{2} \leq 1$. We can therefore now write

$$
\begin{aligned}
\mathbf{K}(w)=\sum_{i=1}^{n} \widehat{f}(i) w_{i} & \leq \sqrt{w_{1}^{2}+w_{2}^{2}} \cdot \sqrt{\widehat{f}^{2}(1)+\widehat{f}^{2}(2)}+\left\|w^{(2)}\right\|_{2} \cdot \sqrt{\sum_{k=3}^{n} \widehat{f}(k)^{2}} \\
& \leq \sqrt{\mathbf{W}^{1}[f]-1 /(256)}+\tau
\end{aligned}
$$

where the first inequality follows by two applications of Cauchy-Schwarz and the second follows by our assumptions. By squaring and expanding, assuming that $\tau>0$ is sufficiently small, we obtain

$$
\begin{aligned}
(\mathbf{K}(w))^{2} & \leq \mathbf{W}^{1}[f]-1 / 300 \\
& \leq \mathbf{W}^{1}[f]-(1 / 300) \mathbf{W}^{1}[f]=(299 / 300) \cdot \mathbf{W}^{1}[f]
\end{aligned}
$$

where the second inequality follows from the fact that $\mathbf{W}^{1}[f] \leq 1$. This proves Lemma 40 . 
Theorem 3 follows easily from Lemma 40 in this subcase using the "basic" Khintchine inequality with optimal constant, $(\mathbf{K}(w))^{2} \geq 1 / 2$. We turn now to the remaining subcase:

(b) $\left(\mathbf{E}_{y}\left[f_{01}(y)\right]>\lambda=3 / 4\right)$ In this case, we show that the value $\widehat{f}(1)$ is so large that it alone causes $\mathbf{W} \leq 1[f]$ to be significantly larger than $1 / 2$. Since $\mathbf{E}_{y}\left[f_{01}(y)\right]>3 / 4$ it must certainly also be the case that $\mathbf{E}_{y}\left[f_{00}(y)\right]>3 / 4$, and by symmetry $\mathbf{E}_{y}\left[f_{10}(y)\right]<-3 / 4$ and $\mathbf{E}_{y}\left[f_{11}(y)<-3 / 4\right.$. Consequently we have $\widehat{f}(1)=\mathbf{E}_{x}\left[f(x) x_{1}\right]>3 / 4$, and so $\mathbf{W}^{\leq 1}[f] \geq \widehat{f}(1)^{2} \geq 9 / 16$.

This concludes the proof of Theorem 3

\section{Alternate proof of Theorem 3}

Recall that it suffices to prove the theorem for balanced LTFs. The idea of the second proof is to perturb the original halfspace slightly so that the perturbed halfspace is defined by a sufficiently anti-concentrated linear form $w^{\prime} \cdot x$. If the perturbed halfspace is regular, one can show that its degree- 1 Fourier weight is close to $2 / \pi$. Otherwise, there exists a large weight, hence an influential variable $x_{1}$ (say). We are then able to show a non-trivial upper bound on the influence of $x_{1}$ on the function $\ell(x)=\left|w^{\prime} \cdot x\right|$.

We require the following terminology:

Definition 42. The (relative) Hamming distance between two Boolean functions $f, g:\{-1,1\}^{n} \rightarrow\{-1,1\}$ is defined as follows: $\operatorname{dist}(f, g) \stackrel{\text { def }}{=} \mathbf{P r}_{x}[f(x) \neq g(x)]$. If $\operatorname{dist}(f, g) \leq \epsilon$ we say that $f$ and $g$ are $\epsilon$-close.

Definition 43. Let $f:\{-1,1\}^{n} \rightarrow\{-1,1\}$ be an LTF, $f(x)=\operatorname{sign}\left(w_{0}+\sum_{i=1}^{n} w_{i} x_{i}\right)$, where the weights are scaled so that $\sum_{i=0}^{n} w_{i}^{2}=1$. Given a particular input $x \in\{-1,1\}^{n}$ we define $\operatorname{marg}(f, x)=\mid w_{0}+$ $\sum_{i=1}^{n} w_{i} x_{i} \mid$.

We start by recalling the following result from [OS11] which essentially says that any LTF is extremely close to another LTF for which almost all points have large margin:

Theorem 44. [Theorem 6.1 in [OS11]] Let $f:\{-1,1\}^{n} \rightarrow\{-1,1\}$ be any LTF and let $0<\tau<1 / 2$. Then there is an LTF $f^{\prime}:\{-1,1\}^{n} \rightarrow\{-1,1\}$ with $\operatorname{dist}\left(f, f^{\prime}\right) \leq \eta(\tau)$ satisfying $\operatorname{Pr}_{x}\left[\operatorname{marg}\left(f^{\prime}, x\right) \leq \kappa(\tau)\right] \leq \tau$, where $\kappa(\tau)=2^{-O\left(\log ^{3}(1 / \tau) / \tau^{2}\right)}$ and $\eta(\tau)=2^{-1 / \kappa(\tau)}$.

Let $0<\tau<\delta$ be sufficiently small universal constants (to be chosen later). Given any balanced LTF $f(x)=\operatorname{sign}(w \cdot x)$, we consider the LTF $f^{\prime}=\operatorname{sign}\left(w^{\prime} \cdot x\right),\left\|w^{\prime}\right\|_{2}=1$, obtained from Theorem 44, so $\operatorname{dist}\left(f, f^{\prime}\right) \leq \eta(\tau)$ and $\operatorname{Pr}_{x}\left[\left|w^{\prime} \cdot x\right| \leq \kappa(\tau)\right] \leq \tau$. We will exploit the anti-concentration of $w^{\prime} \cdot x$ to establish the theorem for $f^{\prime}$. We will then use the fact that $f$ and $f^{\prime}$ are close in Hamming distance to complete the theorem.

We apply Fact 20 for the degree-1 Fourier vectors of $f$ and $f^{\prime}$, i.e. $a_{i}=\widehat{f}(i)$ and $b_{i}=\widehat{f}^{\prime}(i), i \in[n]$. Note that Parseval's identity gives that $\sum_{i=1}^{n}(\widehat{f}(i))^{2} \leq 1$ and $\sum_{i=1}^{n}\left(\widehat{f}^{\prime}(i)\right)^{2} \leq 1$. Moreover, Plancherel's identity implies that

$$
\sum_{i=1}^{n}\left(\widehat{f}(i)-\widehat{f}^{\prime}(i)\right)^{2} \leq \sum_{S \subseteq[n]}\left(\widehat{f}(S)-\widehat{f}^{\prime}(S)\right)^{2}=\mathbf{E}_{x}\left[\left(f(x)-f^{\prime}(x)\right)^{2}\right]=4 \operatorname{dist}\left(f, f^{\prime}\right) \leq 4 \eta .
$$

Therefore,

Therefore, Fact 20 gives that

$$
\left|\mathbf{W}^{1}[f]-\mathbf{W}^{1}\left[f^{\prime}\right]\right| \leq 4 \sqrt{\eta}
$$

The above equation implies that if we show the theorem for $f^{\prime}$ we are done as long as $\eta$ is sufficiently small. We can guarantee this by making $\tau$ sufficiently small. To show the theorem for $f^{\prime}$, we consider two possibilities depending on whether the vector $w^{\prime}$ defining $f^{\prime}$ is $\delta$-regular (where $\delta$ will be determined later).

[Case I: $w^{\prime}$ is $\delta$-regular] In this case, we use the following result from [MORS10]: 
Theorem 45 (Theorem 48 in [MORS10]). Let $\delta>0$ be a sufficiently small universal constant and $f$ be $a$ $\delta$-regular LTF. Then $\left|\mathbf{W}^{1}[f]-W(\mathbf{E}[f])\right| \leq \delta^{1 / 6}$.

We give a full description of the $W(\cdot)$ function in Section 6.1 here we only will use the fact that $W(0)=2 / \pi$. Theorem 45 thus gives that $\mathbf{W}^{1}\left[f^{\prime}\right] \geq \frac{2}{\pi}-\delta^{1 / 6}$ and by (10) we obtain

$$
\mathbf{W}^{1}[f] \geq \frac{2}{\pi}-\delta^{1 / 6}-4 \sqrt{\eta} .
$$

This quantity can be made arbitrarily close to $2 / \pi$ by selecting $\delta, \tau$ to be small enough constants.

[Case II: $w^{\prime}$ is not $\delta$-regular] In this case, we have that $\left|w_{1}^{\prime}\right|=\max _{i}\left|w_{i}^{\prime}\right|>\delta$. Let us assume without loss of generality that $w_{1}^{\prime}>0$. (The other case is entirely similar.) By Proposition 8 and Fact 23 we have

$$
\mathbf{W}^{1}\left[f^{\prime}\right] \geq\left(\mathbf{K}\left(w^{\prime}\right)\right)^{2}=1-\operatorname{Var}(\ell) \geq 1-(1 / 2) \cdot \operatorname{Inf}(\ell)
$$

where $\ell(x)=\left|w^{\prime} \cdot x\right|$. Lemma 24 already implies that $\operatorname{Var}[\ell] \leq 1 / 2$, but we are able to prove a better upper bound in this case. To prove a better upper bound on the variance, we exploit that $w_{1}^{\prime}>\delta$ to upper bound $\operatorname{Inf}_{1}(\ell)$ by a quantity strictly smaller than $\left(w_{1}^{\prime}\right)^{2}$. For this, we recall the following result from [MORS10]:

Theorem 46 (Theorem 39 in [MORS10]). Let $f(x)=\operatorname{sign}\left(\sum_{i=1}^{n} w_{i} x_{i}-w_{0}\right)$ be an LTF such that $\sum_{i} w_{i}^{2}=$ 1 and $\delta \stackrel{\text { def }}{=}\left|w_{1}\right| \geq\left|w_{i}\right|$ for all $i \in[n]$. Let $0 \leq \epsilon \leq 1$ be such that $|\mathbf{E}[f]| \leq 1-\epsilon$. Then $|\hat{f}(1)|=$ $\Omega\left(\delta \epsilon^{6} \log (1 / \epsilon)\right)$.

We can now state and prove our main lemma for this case:

Lemma 47. In the context of Case II, we have $\operatorname{Inf}_{1}(\ell) \leq\left(w_{1}^{\prime}\right)^{2}-2\left(\widehat{f}^{\prime}(1)-2 \tau\right) \kappa(\tau) w_{1}^{\prime}+\left(\widehat{f}^{\prime}(1)-2 \tau\right) \kappa(\tau)^{2}$.

Proof. Since $w_{1}^{\prime}>\delta$ and $\sum_{i=1}^{n}\left(w_{i}^{\prime}\right)^{2}=1$, an application of Theorem 46 gives $\operatorname{Inf}_{1}\left(f^{\prime}\right)=\widehat{f}^{\prime}(1)>c_{1} \cdot w_{1}^{\prime}$, where $c_{1}$ is a universal constant.

To analyze the desired quantity, we partition the hypercube $\{-1,1\}^{n}$ into pairs $\left(x^{+}, x^{-}\right)$that differ only in the fist coordinate with $x_{1}^{+}=1$ and $x_{1}^{-}=-1$. That is $x^{+}=(1, y)$ and $x^{-}=(-1, y)$ with $y \in$ $\{-1,1\}^{n-1}$. We say that such a pair is "good" if both the following conditions hold: (1) the corresponding hypercube edge is bi-chromatic (i.e. $f^{\prime}\left(x^{+}\right)=1$ and $\left.f^{\prime}\left(x^{-}\right)=-1\right)$, and (2) $\min \left\{\left|w^{\prime} \cdot x^{+}\right|,\left|w^{\prime} \cdot x^{-}\right|\right\} \geq$ $\kappa(\tau)$. It is easy to see that the fraction of pairs that are "good" is at least $\widehat{f}^{\prime}(1)-2 \tau$, i.e. $\mathbf{P r}_{y \in\{-1,1\}^{n-1}}[\mathcal{G}] \geq$ $\widehat{f}^{\prime}(1)-2 \tau$, where $\mathcal{G}$ is the event $\mathcal{G}=\left\{y \in\{-1,1\}^{n-1} \mid \quad\right.$ the pair $(1, y),(-1, y)$ is good $\}$. Indeed, the probability that the edge $(1, y),(-1, y)$ is monochromatic is $1-\mathbf{I n f}_{1}\left(f^{\prime}\right)=1-\hat{f}^{\prime}(1)$ and the probability that either $\left|w^{\prime} \cdot x^{+}\right| \leq \kappa(\tau)$ or $\left|w^{\prime} \cdot x^{-}\right| \leq \kappa(\tau)$ is at most $\tau$, hence the claim follows by a union bound.

Now if $y \in\{-1,1\}^{n-1}$ is such that the corresponding pair $x^{+}=(1, y)$ and $x^{-}=(-1, y)$ is good, we have that $\left|w^{\prime} \cdot x^{+}\right|=w_{1}^{\prime}+c^{\prime} \geq \kappa(\tau)$ and $\left|w^{\prime} \cdot x^{-}\right|=w_{1}^{\prime}-c^{\prime} \geq \kappa(\tau)$, where $c^{\prime}=\left(w_{2}^{\prime}, \ldots, w_{n}^{\prime}\right) \cdot y$. From this we deduce that $\left|c^{\prime}\right| \leq\left|w_{1}^{\prime}-\kappa(\tau)\right| \leq\left|w_{1}^{\prime}\right|$, where the second inequality holds for a sufficiently small choice of $\tau$. Hence, the analysis of Lemma24yields that in this case $\operatorname{Var}\left[\ell\left(x_{1}, y\right)\right]=c^{\prime 2} \leq\left(w_{1}^{\prime}-\kappa(\tau)\right)^{2}$. In all other cases, Lemma24yields the upper bound $\operatorname{Var}\left[\ell\left(x_{1}, y\right)\right]=c^{\prime 2} \leq\left(w_{1}^{\prime}\right)^{2}$. We can thus bound from above the desired influence as follows:

$$
\begin{aligned}
\operatorname{Inf}_{1}(\ell) & =\mathbf{E}_{y \in\{-1,1\}^{n-1}}\left[\operatorname{Var}\left[\ell\left(x_{1}, y\right)\right]\right] \\
& \leq\left(\widehat{f}^{\prime}(1)-2 \tau\right) \cdot\left(w_{1}^{\prime}-\kappa(\tau)\right)^{2}+\left(1-\widehat{f}^{\prime}(1)+2 \tau\right)\left(w_{1}^{\prime}\right)^{2} \\
& \leq\left(w_{1}^{\prime}\right)^{2}-2\left(\widehat{f}^{\prime}(1)-2 \tau\right) \kappa(\tau) w_{1}^{\prime}+\left(\widehat{f}^{\prime}(1)-2 \tau\right) \kappa(\tau)^{2} .
\end{aligned}
$$

This completes the proof.

\footnotetext{
${ }^{2}$ This is the only possibility since $w_{1}^{\prime}>0$, hence $f^{\prime}$ is monotone nondecreasing in $x_{1}$.
} 
Combining Lemma47 with our earlier arguments, we obtain

$$
\mathbf{W}^{1}\left[f^{\prime}\right] \geq \frac{1}{2}+\left(\widehat{f}^{\prime}(1)-2 \tau\right) \kappa(\tau) w_{1}^{\prime}-\frac{\left(\widehat{f}^{\prime}(1)-2 \tau\right) \kappa(\tau)^{2}}{2}
$$

and using (10) we conclude

$$
\mathbf{W}^{1}[f] \geq \frac{1}{2}+\left(\widehat{f}^{\prime}(1)-2 \tau\right) \kappa(\tau) w_{1}^{\prime}-\frac{\left(\widehat{f}^{\prime}(1)-2 \tau\right) \kappa(\tau)^{2}}{2}-4 \sqrt{\eta(\tau)} .
$$

At this point it is straightforward to complete the proof of Theorem 3 Indeed, we select $\delta>0$ to be a sufficiently small constant and $\tau \stackrel{\text { def }}{=} c_{1} \cdot \delta / 4 \ll \delta$. First, note that the bound of (11) for the regular case can be made arbitrarily close to $2 / \pi$. Regarding the bound of (12) for the non-regular case observe that

$$
\widehat{f}^{\prime}(1)-2 \tau>c_{1} \delta-c_{1} \delta / 2=c_{1} \delta / 2
$$

which means that the advantage over $1 / 2$ is at least

$$
(1 / 2) \cdot c_{1} \delta^{2} \kappa(\tau)-(1 / 4) \cdot c_{1} \delta \kappa(\tau)^{2}-4 \sqrt{\eta(\tau)}
$$

which is lower bounded by a universal positive constant, since the second and the third terms are negligible compared to the first for our choice of parameters. This concludes the proof of Theorem 3

\section{Proof of Theorem 4: An approximation algorithm for $\mathbf{W}^{\leq 1}[\mathrm{LTF}]$}

Our approach heavily uses Gaussian analysis, so we record some basic definitions and facts that we will need below.

\subsection{Gaussian Facts}

Definition 48. We write $\phi$ for the probability density function of a standard (i.e. zero mean, unit variance) Gaussian; i.e. $\phi(t)=(2 \pi)^{-1 / 2} e^{-t^{2} / 2}$. We denote by $\mathcal{N}(0,1)$ the corresponding distribution and by $\mathcal{N}(0,1)^{n}\left(\right.$ or $\left.\mathcal{N}^{n}\right)$ the standard n-dimensional Gaussian distribution.

Fact 49. (Rotational Invariance) Let $U: \mathbb{R}^{n} \rightarrow \mathbb{R}^{n}$ be a unitary transformation, i.e., $U^{t} U=I$. If $x \sim \mathcal{N}(0,1)^{n}$, then $U x \sim \mathcal{N}(0,1)^{n}$.

Definition 50. Let $h_{\theta}: \mathbb{R} \rightarrow\{-1,1\}$ denote the function of one Gaussian random variable $x$ given by $h_{\theta}(x)=\operatorname{sign}(x-\theta)$.

Definition 51. The function $\mu: \mathbb{R} \cup\{ \pm \infty\} \rightarrow[-1,1]$ is defined as $\mu(\theta)=\mathbf{E}_{x \sim \mathcal{N}(0,1)}\left[h_{\theta}(x)\right]$. Explicitly, $\mu(\theta)=-1+2 \int_{\theta}^{\infty} \phi(x) d x$. We note that $\mu$ is strictly monotone decreasing, hence invertible on $[-1,1]$.

Definition 52. The function $W:[-1,1] \rightarrow[0,2 / \pi]$ is defined by $W(x)=\left(2 \phi\left(\mu^{-1}(x)\right)\right)^{2}$. Equivalently, $W$ is defined so that $W(\mu(\theta))=(2 \phi(\theta))^{2}$.

The next two facts appear as Propositions 24 and 25 in [MORS10] respectively.

Fact 53. Let $X \sim \mathcal{N}(0,1)$. We have:

- (i) $\mathbf{E}[|X-\theta|]=2 \phi(\theta)-\theta \mu(\theta)$,

- (ii) $\left|\mu^{\prime}\right| \leq \sqrt{2 / \pi}$ everywhere and $\left|W^{\prime}\right|<1$ everywhere, and 
- (iii) If $|\nu|=1-\eta$, then $W(\nu)=\Theta\left(\eta^{2} \log (1 / \eta)\right)$.

Fact 54. Let $f(x)=\operatorname{sign}(w \cdot x-\theta)$ be an LTF such that $\|w\|_{2}=1$. Then

- (i) $\tilde{f}(0) \stackrel{\text { def }}{=} \mathbf{E}_{x \sim \mathcal{N}^{n}}[f(x)]=\mu(\theta)$,

- (ii) $\tilde{f}(i) \stackrel{\text { def }}{=} \mathbf{E}_{x \sim \mathcal{N}^{n}}\left[f(x) x_{i}\right]=\sqrt{W(\tilde{f}(0))} w_{i}$, for all $i \in[n]$, and

- (iii) $\sum_{i=1}^{n} \tilde{f}^{2}(i)=W(\tilde{f}(0))$.

\subsection{Proof of Theorem 4}

We recall the statement of Theorem 4 :

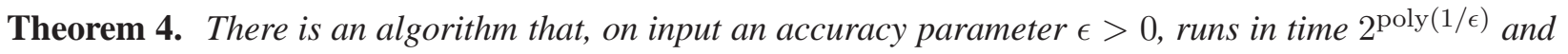
outputs a value $\Gamma_{\epsilon}$ such that

$$
\mathbf{W}^{\leq 1}[\mathbf{L T F}] \leq \Gamma_{\epsilon} \leq \mathbf{W}^{\leq 1}[\mathbf{L T F}]+\epsilon .
$$

We recall the simple algorithm used to prove Theorem 4 from Section 1.4

Let $K=\Theta\left(\epsilon^{-24}\right)$. Enumerate all $K$-variable zero-threshold LTFs, and output the value

$$
\Gamma_{\epsilon} \stackrel{\text { def }}{=} \min \left\{\mathbf{W}^{1}[f]: f \text { is a zero-threshold } K \text {-variable LTF. }\right\} .
$$

As described in Section 1.4 it suffices to prove that for any zero-threshold $n$-variable LTF $f(x)=$ $\operatorname{sign}(w \cdot x)$, there is a $K$-variable zero-threshold LTF $g$, where $K=\Theta\left(\epsilon^{-24}\right)$, such that

$$
\left|\mathbf{W}^{1}[f]-\mathbf{W}^{1}[g]\right|<\epsilon ;
$$

we now proceed with the proof. We can of course assume that $n>K$, since otherwise (13) is trivially satisfied for $g=f$ with $\epsilon=0$.

We choose a parameter $\delta=O\left(\epsilon^{6}\right)$; as described in Section 1.4 the proof is by case analysis on the value of the $\delta$-critical index $c(w, \delta)$ of the weight vector $w$. Consider a parameter $L=L(\delta)=\tilde{\Theta}\left(\delta^{-2}\right)$. We consider the following two cases:

[Case I: Large critical index, i.e. $c(w, \delta) \geq L(\delta)$ ] In this case, the proof follows easily from the following lemma:

Lemma 55 (Case II(a) of Theorem 1 of [Ser07]). Let $f(x)=\operatorname{sign}(w \cdot x)=\operatorname{sign}\left(w_{H} \cdot x_{H}+w_{T} \cdot x_{T}\right)$, where $w$ is proper, $H=[L(\delta)]$ and $T=[n] \backslash H$. If $c(w, \delta) \geq L(\delta)$, then $f$ is $\delta$-close in Hamming distance to function the junta $g(x)=\operatorname{sign}\left(w_{H} \cdot x_{H}\right)$.

Since $\operatorname{dist}(f, g) \leq \delta$, Fact 20 implies that $\left|\mathbf{W}^{1}[f]-\mathbf{W}^{1}[g]\right|<4 \sqrt{\delta}<\epsilon$. Noting that $g$ is a zerothreshold $K$-variable LTF (since $L<K$ ) completes the proof of Case I.

[Case II: Small critical index, i.e. $c(w, \delta)<L(\delta)$ ] This case requires an elaborate analysis: at a high-level we apply a variable reduction technique to obtain a junta $g$ that closely approximates the degree- 1 Fourier weight of $f$. Note that there is no guarantee (and it is typically not the case) that $f$ and $g$ are close in Hamming distance. Formally, we prove the following theorem: 
Theorem 56. Let $f(x)=\operatorname{sign}(w \cdot x)=\operatorname{sign}\left(w_{H} \cdot x_{H}+w_{T} \cdot x_{T}\right)$, where $w$ is proper, $H=[c(w, \delta)]$ and $T=[n] \backslash H$. Consider the LTF $g:\{-1,1\}^{|H|+M} \rightarrow\{-1,1\}$, with $M=\Theta\left(\epsilon^{-24}\right)$, defined by

$$
g\left(x_{H}, z\right)=\operatorname{sign}\left(w_{H} x_{H}+\left\|w_{T}\right\|_{2} \cdot \sum_{i=1}^{M} \frac{z_{i}}{\sqrt{M}}\right) .
$$

Then $\left|\mathbf{W}^{1}[f]-\mathbf{W}^{1}[g]\right|<\epsilon$.

Note that $g$ depends on $|H|+M \leq L+M \leq K$ variables. Hence, Theorem 56 completes the analysis of Case II. We refer the reader to Section 1.4 for intuition and motivation behind Theorem 56 and proceed to its proof in the next subsection.

\subsection{Proof of Theorem 56}

Let $f:\{-1,1\}^{n} \rightarrow\{-1,1\}$ where $f(x)=\operatorname{sign}(w \cdot x)=\operatorname{sign}\left(w_{H} x_{H}+w_{T} x_{T}\right)$, where the tail vector $w_{T}$ is $\delta$-regular. Assume wlog that $\left\|w_{T}\right\|_{2}=1$. We proceed in the following three steps, which together yield Theorem 56 .

Step 1: "Gaussianizing" the tail. First some notation: we write $\mathcal{U}_{n}$ to denote the uniform distribution over $\{-1,1\}^{n}$. Our main result in this case is the following theorem, which roughly says that letting tail variables take Gaussian rather than Boolean values does not change the "degree-1 Fourier coefficients" by much:

Theorem 57. Let $f=\operatorname{sign}\left(w_{H} \cdot x_{H}+w_{T} \cdot x_{T}\right)$. For $i \in[n]$ define $\widehat{f}(i)=\mathbf{E}_{x \sim \mathcal{U}_{n}}\left[f(x) x_{i}\right]$ and $\tilde{f}(i)=$ $\mathbf{E}_{x_{H} \sim \mathcal{U}_{|H|}, x_{T} \sim \mathcal{N}|T|}\left[f(x) x_{i}\right]$. If $w_{T}$ is $\tau$-regular then

$$
\sum_{i=1}^{n}(\widehat{f}(i)-\tilde{f}(i))^{2}=O\left(\tau^{1 / 6}\right)
$$

Note that by applying Fact 20 the above theorem implies that $\mathbf{W}^{1}[f] \approx^{\tau^{1 / 12}} \widetilde{\mathbf{W}^{1}}[f]$, where we define $\widetilde{\mathbf{W}^{1}}[f] \stackrel{\text { def }}{=} \sum_{i=1}^{n}(\tilde{f}(i))^{2}$.

To prove Theorem 57 we need a few lemmas. Our first lemma shows that for a regular LTF, its degree-1 Fourier coefficients are close to its corresponding Hermite coefficients.

Lemma 58. Let $f(x)=\operatorname{sign}\left(w \cdot x-w_{0}\right)$ be an LTF. For $i \in[n]$ define $\widehat{f}(i) \stackrel{\text { def }}{=} \mathbf{E}_{x \in \mathcal{U}_{n}}\left[f(x) x_{i}\right]$ and $\tilde{f}(i) \stackrel{\text { def }}{=} \mathbf{E}_{x \in \mathcal{N}^{n}}\left[f(x) x_{i}\right]$. If $w$ is $\tau$-regular, then $\sum_{i=1}^{n}(\widehat{f}(i)-\tilde{f}(i))^{2}=O\left(\tau^{1 / 6}\right)$.

Proof. We can assume that $\|w\|_{2}=1$. Since $w$ is $\tau$-regular, by Fact 19 (i) we have that $\widehat{f}(0) \approx^{\tau} \tilde{f}(0)$. It suffices to show that

$$
\sum_{i=1}^{n} \widehat{f}(i)^{2}+\sum_{i=1}^{n} \tilde{f}(i)^{2} \approx^{\tau^{1 / 6}} 2 \sum_{i=1}^{n} \widehat{f}(i) \tilde{f}(i)
$$

We first note that the lemma follows easily for the case that $\left|w_{0}\right|>\sqrt{2 \ln (2 / \tau)}$. In this case, by an application of the Hoeffding bound (Theorem $[15)$ it follows that $|\widehat{f}(0)| \geq 1-2 \tau$, hence $|\tilde{f}(0)| \geq 1-3 \tau$. By Parseval's identity we have

$$
\sum_{i=1}^{n} \widehat{f}(i)^{2} \leq \sum_{\emptyset \neq S \subseteq[n]} \widehat{f}(S)^{2}=1-\widehat{f}(0)^{2} \leq 4 \tau ;
$$

similarly, in the Gaussian setting, we get

$$
\sum_{i=1}^{n} \tilde{f}(i)^{2} \leq \sum_{\emptyset \neq S} \tilde{f}(S)^{2} \leq 1-\tilde{f}(0)^{2} \leq 6 \tau .
$$


Hence, we conclude that $\sum_{i=1}^{n}(\widehat{f}(i)-\tilde{f}(i))^{2} \leq 2 \sum_{i=1}^{n} \widehat{f}(i)^{2}+2 \sum_{i=1}^{n} \tilde{f}(i)^{2}=O(\tau)$.

We now consider the case that $\left|w_{0}\right| \leq \sqrt{2 \ln (2 / \tau)}$ and proceed to prove (14). By Fact 54(iii) we get $\sum_{i=1}^{n} \tilde{f}(i)^{2}=W(\tilde{f}(0))$. Moreover, Theorem 45 gives that $\sum_{i=1}^{n} \widehat{f}(i)^{2} \approx^{\tau^{1 / 6}} W(\widehat{f}(0))$. We now claim that $W(\widehat{f}(0)) \approx^{\tau} W(\tilde{f}(0))$. This follows from the mean value theorem, since $\widehat{f}(0) \approx^{\tau} \tilde{f}(0)$ and $\left|W^{\prime}\right|<1$ everywhere, by Fact53. Therefore, we conclude that the LHS of (14) satisfies

$$
\sum_{i=1}^{n} \widehat{f}(i)^{2}+\sum_{i=1}^{n} \tilde{f}(i)^{2} \approx^{\tau^{1 / 6}} 2 W(\tilde{f}(0))
$$

For the RHS of (14) we can write

$$
\sum_{i=1}^{n} \widehat{f}(i) \tilde{f}(i)=\sqrt{W(\tilde{f}(0))} \sum_{i=1}^{n} w_{i} \widehat{f}(i)=\sqrt{W(\tilde{f}(0))} \mathbf{E}_{x \in \mathcal{U}_{n}}\left[(w \cdot x) \operatorname{sign}\left(w \cdot x-w_{0}\right)\right]
$$

where the first equation follows from Fact 54 (ii) and the third is Plancherel's identity. Moreover, by definition we have

$$
\mathbf{E}_{x \in \mathcal{U}_{n}}\left[(w \cdot x) \operatorname{sign}\left(w \cdot x-w_{0}\right)\right]=\mathbf{E}_{x \in \mathcal{U}_{n}}\left[\left|w \cdot x-w_{0}\right|\right]+w_{0} \mathbf{E}_{x \in \mathcal{U}_{n}}[f(x)] .
$$

Recalling Fact 19 we deduce that

$$
\mathbf{E}_{x \in \mathcal{U}_{n}}\left[(w \cdot x) \operatorname{sign}\left(w \cdot x-w_{0}\right)\right] \approx^{\left(\left|w_{0}\right|+1\right) \tau} \mathbf{E}_{x \in \mathcal{N}^{n}}\left[(w \cdot x) \operatorname{sign}\left(w \cdot x-w_{0}\right)\right] .
$$

Now, the RHS above satisfies

$$
\begin{aligned}
\mathbf{E}_{x \in \mathcal{N}^{n}}\left[(w \cdot x) \operatorname{sign}\left(w \cdot x-w_{0}\right)\right] & =\mathbf{E}_{X \in \mathcal{N}}\left[\left|X-w_{0}\right|\right]+w_{0} \mathbf{E}_{x \in \mathcal{N}^{n}}[f(x)] \\
& =2 \phi\left(w_{0}\right)=\sqrt{W(\tilde{f}(0))}
\end{aligned}
$$

where the first equality follows by definition, the second uses Fact53(i) and the third uses the definition of $\phi$. Therefore,

$$
\mathbf{E}_{x \in \mathcal{U}_{n}}\left[(w \cdot x) \operatorname{sign}\left(w \cdot x-w_{0}\right)\right] \approx^{\left(w_{0}+1\right) \tau} \sqrt{W(\tilde{f}(0))}
$$

Since the function $W$ is uniformly bounded from above by $2 / \pi$, we conclude that the RHS of (14) satisfies

$$
\sum_{i=1}^{n} \widehat{f}(i) \tilde{f}(i) \approx^{\left(\left|w_{0}\right|+1\right) \tau} W(\tilde{f}(0))
$$

The proof now follows from the fact that $\left(\left|w_{0}\right|+1\right) \tau<\tau^{1 / 6}$, which holds since $\left|w_{0}\right|=O(\sqrt{\log (1 / \tau)})$.

Our next lemma, a simple generalization of Lemma 58 above, shows that for any LTF, if the variables in its tail are replaced by independent standard Gaussians, the corresponding degree 1-Fourier and Hermite coefficients of the tail variables are very close to each other.

Lemma 59. Let $f=\operatorname{sign}\left(w_{H} \cdot x_{H}+w_{T} \cdot x_{T}\right)$. For $i \in T$, define $\widehat{f}(i)=\mathbf{E}_{x \sim \mathcal{U}_{n}}\left[f(x) x_{i}\right]$ and $\tilde{f}(i)=$ $\mathbf{E}_{x_{H} \sim \mathcal{U}_{|H|}, x_{T} \sim \mathcal{N}|T|}\left[f(x) x_{i}\right]$. If $w_{T}$ is $\tau$-regular, then we have $\sum_{i \in T}(\widehat{f}(i)-\tilde{f}(i))^{2}=O\left(\tau^{1 / 6}\right)$.

Proof. Fix an assignment $\rho \in\{-1,1\}^{|H|}$ to the variables in $H$ (head coordinates) and consider the restriction $f_{\rho}$ over the coordinates in $T$, i.e. $f_{\rho}\left(x_{T}\right)=\operatorname{sign}\left(w_{H} \cdot \rho+w_{T} \cdot x_{T}\right)$. For every assignment $\rho$, the restriction $f_{\rho}$ is a $\tau$-regular LTF (with a different threshold); hence Lemma 58 yields that for all $\rho \in\{-1,1\}^{|H|}$ we have

$$
\sum_{i \in T}\left(\widehat{f}_{\rho}(i)-\tilde{f}_{\rho}(i)\right)^{2}=O\left(\tau^{1 / 6}\right)
$$


Hence, we obtain

$$
\begin{aligned}
\sum_{i \in T}(\widehat{f}(i)-\tilde{f}(i))^{2}=\sum_{i \in T}\left(\mathbf{E}_{\rho \sim \mathcal{U}_{|H|}}\left[\widehat{f}_{\rho}(i)-\tilde{f}_{\rho}(i)\right]\right)^{2} & \leq \sum_{i \in T} \mathbf{E}_{\rho \sim \mathcal{U}_{|H|}}\left[\left(\widehat{f}_{\rho}(i)-\tilde{f}_{\rho}(i)\right)^{2}\right] \\
& =\mathbf{E}_{\rho \sim \mathcal{U}_{|H|}}\left[\sum_{i \in T}\left(\widehat{f}_{\rho}(i)-\tilde{f}_{\rho}(i)\right)^{2}\right] \\
& =O\left(\tau^{1 / 6}\right)
\end{aligned}
$$

where the first equality uses the definition of the Fourier/Hermite coefficients, the first inequality follows from Jensen's inequality for each summand, the second equality follows by linearity and the last equality uses (15).

Replacing the Boolean tail variables by Gaussians alters the Fourier coefficients of the head variables as well. Our next lemma shows that the corresponding change is bounded in terms of the regularity of the tail.

Lemma 60. Let $f=\operatorname{sign}\left(w_{H} \cdot x_{H}+w_{T} \cdot x_{T}\right)$. For $i \in H$ define $\widehat{f}(i)=\mathbf{E}_{x \sim \mathcal{U}_{n}}\left[f(x) x_{i}\right]$ and $\tilde{f}(i)=$ $\mathbf{E}_{x_{H} \sim \mathcal{U}_{|H|}, x_{T} \sim \mathcal{N}|T|}\left[f(x) x_{i}\right]$. If $w_{T}$ is $\tau$-regular, then we have $\sum_{i \in H}(\widehat{f}(i)-\tilde{f}(i))^{2}=O\left(\tau^{2}\right)$.

Proof. We define the functions $f^{\prime}:\{-1,1\}^{|H|} \rightarrow[-1,1]$ and $f^{\prime \prime}:\{-1,1\}^{|H|} \rightarrow[-1,1]$ as follows :

$$
f^{\prime}\left(x_{H}\right)=\mathbf{E}_{x_{T} \in \mathcal{U}_{|T|}}\left[f\left(x_{H}, x_{T}\right)\right] \text { and } f^{\prime \prime}\left(x_{H}\right)=\mathbf{E}_{x_{T} \in \mathcal{N}|T|}\left[f\left(x_{H}, x_{T}\right)\right] .
$$

By definition, for all $i \in H$ it holds $\widehat{f}^{\prime}(i)=\widehat{f}(i)$ and $\widehat{f^{\prime \prime}}(i)=\tilde{f}(i)$. We can therefore write

$$
\begin{aligned}
\sum_{i \in H}(\widehat{f}(i)-\tilde{f}(i))^{2} & =\sum_{i \in H}\left(\widehat{f}^{\prime}(i)-\widehat{f^{\prime \prime}}(i)\right)^{2} \\
& \leq \sum_{S \subseteq H}\left(\widehat{f}^{\prime}(S)-\widehat{f^{\prime \prime}}(S)\right)^{2}=\mathbf{E}_{x \in \mathcal{U}_{|H|}}\left(f^{\prime}(x)-f^{\prime \prime}(x)\right)^{2} \leq\left\|f^{\prime}-f^{\prime \prime}\right\|_{\infty}^{2}
\end{aligned}
$$

where the second equality is Parseval's identity and the final inequality follows from the monotonicity of the norms $\left(\|\cdot\|_{\infty}\right.$ denotes the sup-norm of a random variable).

In order to bound $\left\|f^{\prime}-f^{\prime \prime}\right\|_{\infty}$ we exploit the regularity of the tail via the Berry-Esséen theorem. Indeed, fix an assignment $\rho \in\{-1,1\}^{|H|}$ to $x_{H}$. Then

$$
\begin{array}{r}
\left|f^{\prime}(\rho)-f^{\prime \prime}(\rho)\right| \leq 2 \mid \operatorname{Pr}_{x_{T} \in\{-1,1\}|T|}\left[w_{T} \cdot x_{T}+w_{H} \cdot \rho \geq 0\right]- \\
\operatorname{Pr}_{x_{T} \in \mathcal{N}|T|}\left[w_{T} \cdot x_{T}+w_{H} \cdot \rho \geq 0\right] \mid
\end{array}
$$

Since $w_{T}$ is $\tau$-regular, by Fact 17, the RHS above is bounded from above by $2 \tau$. Since this holds for any restriction $\rho$ to the head we conclude that $\left\|f^{\prime}-f^{\prime \prime}\right\|_{\infty} \leq 2 \tau$ as desired.

Theorem 57 follows by combining Lemmas 59 and 60 .

Step 2: "Collapsing" the tail. Let $F:\{-1,1\}^{|H|} \times \mathbb{R} \rightarrow\{-1,1\}$ be defined by $F\left(x_{H}, y\right)=\operatorname{sign}\left(w_{H} x_{H}+\right.$ $y$ ) (recall that we have assumed that $w$ is scaled so that the "tail weight" $\left\|w_{T}\right\|_{2}$ equals 1 ). For $i \in H$, we define

$$
\widehat{F}(i)=\mathbf{E}_{x_{H} \sim \mathcal{U}_{|H|}, y \sim \mathcal{N}(0,1)}\left[F\left(x_{H}, y\right) x_{i}\right]
$$

and

$$
\widehat{F}(y)=\mathbf{E}_{x_{H} \sim \mathcal{U}_{|H|}, y \sim \mathcal{N}(0,1)}\left[F\left(x_{H}, y\right) y\right] .
$$

We also denote $\widetilde{\mathbf{W}^{1}}[F]=\sum_{i \in H}(\widehat{F}(i))^{2}+\widehat{F}(y)^{2}$. Our main result for this step is that "collapsing" all $|T|$ tail Gaussian variables to a single Gaussian variable does not change the degree-1 "Fourier weight": 
Theorem 61. We have that $\widetilde{\mathbf{W}^{1}}[F]=\widetilde{\mathbf{W}^{1}}[f]$.

The theorem follows by combining the following two lemmas.

Lemma 62. For every $i \in H, \tilde{f}(i)=\widehat{F}(i)$.

Proof. The lemma follows straightforwardly by the definitions. Indeed, for every $i \in H$,

$$
\begin{aligned}
\tilde{f}(i) & =\mathbf{E}_{x_{H} \sim \mathcal{U}_{|H|}, x_{T} \sim \mathcal{N}|T|}\left[\operatorname{sign}\left(w_{H} x_{H}+w_{T} x_{T}\right) x_{i}\right] \\
& =\mathbf{E}_{x_{H} \sim \mathcal{U}_{|H|}, y \sim \mathcal{N}(0,1)}\left[\operatorname{sign}\left(w_{H} x_{H}+y\right) x_{i}\right]=\widehat{F}(i)
\end{aligned}
$$

where the third equality uses the fact that $w_{T} \cdot x_{T}$ is distributed as $\mathcal{N}(0,1)$.

Lemma 63. We have that $(\widehat{F}(y))^{2}=\sum_{i \in T}(\tilde{f}(i))^{2}$.

Proof. This lemma is intuitively clear but we nonetheless give a proof. We need the following simple propositions.

Proposition 64. Let $h: \mathbb{R}^{m} \rightarrow \mathbb{R}$ with $h \in L_{2}\left(\mathcal{N}(0,1)^{m}\right)$. Let $U: \mathbb{R}^{m} \rightarrow \mathbb{R}^{m}$ be a unitary linear transformation. For $i \in[m]$, define $\tilde{h}(i)=\mathbf{E}_{x \sim \mathcal{N}^{m}}\left[h(x) x_{i}\right]$ and $\tilde{h}(i)^{\prime}=\mathbf{E}_{x \sim \mathcal{N}^{m}}\left[h(x)(U x)_{i}\right]$. Then, $\sum_{i=1}^{m} \tilde{h}(i)^{2}=\sum_{i=1}^{m} \tilde{h}(i)^{\prime 2}$.

Proof. Let $(U x)_{i}=\sum_{j=1}^{m} a_{i j} x_{j}$. By linearity, we get that $\tilde{h}(i)^{\prime}=\sum_{j=1}^{m} a_{i j} \tilde{h}(j)$. Then,

$$
\sum_{i=1}^{m} \tilde{h}(i)^{\prime 2}=\sum_{j=1}^{m}\left(\sum_{i=1}^{m} a_{i j}^{2}\right) \tilde{h}(j)^{2}+\sum_{j \neq i}\left(\sum_{k=1}^{m} a_{k j} a_{k i}\right) \tilde{h}(i) \tilde{h}(j)
$$

By elementary properties of unitary matrices, we have (i) $\sum_{i=1}^{m} a_{i j}^{2}=1$ for all $j$, and (ii) $\sum_{k=1}^{m} a_{k j} a_{k i}=0$ for $i \neq j$. Substitution completes the proof.

Proposition 65. Let $\Psi: \mathbb{R} \rightarrow \mathbb{R}$ and $\Phi: \mathbb{R}^{m} \rightarrow \mathbb{R}$ with $\Phi \in L_{2}\left(\mathcal{N}(0,1)^{m}\right)$ defined as $\Phi(x)=$ $\Psi\left(\sum_{i=1}^{m} w_{i} x_{i}\right)$, where $x, w \in \mathbb{R}^{m}$ with $\|w\|_{2}=1$. Then $\sum_{i=1}^{m} \widetilde{\Phi}\left(x_{i}\right)^{2}=\widetilde{\Psi}(y)^{2}$.

Proof. It is clear there is a unitary matrix $U$ such that $U: x_{1} \mapsto \sum_{i=1}^{m} w_{i} x_{i}$. Hence, an application of Proposition 64 gives us

$$
\sum_{i=1}^{m} \tilde{\Phi}\left(x_{i}\right)^{2}=\sum_{i=1}^{m}\left(\mathbf{E}_{x \in \mathcal{N}^{m}}\left[\Phi(x) \cdot(U x)_{i}\right]\right)^{2}
$$

Now observe that for $i>1, \mathbf{E}_{x \in \mathcal{N}^{m}}\left[\Phi(x) \cdot(U x)_{i}\right]=0$ as $\Phi(x)$ is independent of $(U x)_{i}$. Using the rotational invariance of the Gaussian measure and using $y$ instead of $(U x)_{1}$ we deduce

$$
\mathbf{E}_{x \in \mathcal{N}^{m}}\left[\Phi(x) \cdot(U x)_{1}\right]=\mathbf{E}_{y \in \mathcal{N}(0,1)}[(\Psi(y) y]=\widetilde{\Psi}(y) .
$$

Combining with (16) completes the proof.

The proof of the lemma follows by a simple application of the above proposition. Indeed, set $\Psi(y)=$ $\mathbf{E}_{x_{H} \sim \mathcal{U}_{|H|}}\left[F\left(x_{H}, y\right)\right]$. An application of Proposition 65 gives us $\sum_{i \in T} \widetilde{\Phi}\left(x_{i}\right)^{2}=\widetilde{\Psi}(y)^{2}$. Now note that for $i \in T$, by definition, $\widetilde{\Phi}\left(x_{i}\right)=\widetilde{f}(i)$, and $\widetilde{\Psi}(y)=\widehat{F}(y)$. This completes the proof of the lemma. 
Step 3: "Booleanizing" the tail. Let $M=\Theta\left(\epsilon^{-24}\right)$. Consider the LTF $g$ mapping $\left(x_{H}, z\right) \rightarrow\{-1,1\}$, where $x_{H} \in\{-1,1\}^{|H|}$ and $z \in\{-1,1\}^{M}$, defined by

$$
g\left(x_{H}, z\right)=\operatorname{sign}\left(w_{H} \cdot x_{H}+\left(\sum_{i=1}^{M} z_{i}\right) / \sqrt{M}\right) .
$$

In this step we show that replacing the (single) Gaussian tail variable with a scaled sum of Boolean variables does not change the degree-1 "Fourier weight" by much:

Theorem 66. We have that $\left|\widetilde{\mathbf{W}^{1}}[F]-\mathbf{W}^{1}[g]\right|=O\left(M^{-1 / 24}\right)$.

As expected the theorem follows by combining two lemmas, one to deal with the head and one for the tail.

Lemma 67. We have $\sum_{i \in H}(\widehat{F}(i)-\widehat{g}(i))^{2}=O\left(M^{-1}\right)$.

Proof. The proof closely parallels that of Lemma60. Namely, we will define the function $h, h^{\prime}:\{-1,1\}^{|H|} \rightarrow$ $[-1,1]$ as $h\left(x_{H}\right)=\mathbf{E}_{y \sim \mathcal{N}(0,1)}\left[F\left(x_{H}, y\right)\right]$ and $h^{\prime}\left(x_{H}\right)=\mathbf{E}_{z \sim \mathcal{U}_{M}}\left[g\left(x_{H}, z\right)\right]$. Note that for $i \in H, \widehat{h}(i)=$ $\widehat{F}(i)$ and $\widehat{h}^{\prime}(i)=\widehat{g}(i)$. As in Lemma 60, we have $\sum_{i \in H}\left(\widehat{h}(i)-\widehat{h}^{\prime}(i)\right)^{2} \leq\left\|h-h^{\prime}\right\|_{\infty}^{2}$. For any $\rho \in\{-1,1\}^{|H|}$, we can write

$$
\left|h(\rho)-h^{\prime}(\rho)\right|=2\left|\mathbf{P r}_{y \in \mathcal{N}(0,1)}\left[w_{H} \rho+y \geq 0\right]-\operatorname{Pr}_{z \in \mathcal{U}_{M}}\left[w_{H} \rho+\sum_{i=1}^{M} z_{i} / \sqrt{M} \geq 0\right]\right| .
$$

Theorem 16 shows that the RHS is bounded from above by $2 / \sqrt{M}$, which completes the proof of the lemma.

Lemma 68. Let $F$ and $g$ as defined above. Then $\left|\sum_{i=1}^{M}\left(\widehat{g}\left(z_{i}\right)\right)^{2}-(\widehat{F}(y))^{2}\right|=O\left(M^{-1 / 24}\right)$.

Proof. First note that, by symmetry, for all $i, j \in[M]$ we have $\widehat{g}\left(z_{i}\right)=\widehat{g}\left(z_{j}\right)$. By definition, we can write

$$
\widehat{g}\left(z_{i}\right)=\mathbf{E}_{x_{H}} \mathbf{E}_{z \sim \mathcal{U}_{M}}\left[\operatorname{sign}\left(w_{H} \cdot x_{H}+\left(\sum_{i=1}^{M} z_{i}\right) / \sqrt{M}\right)\right]
$$

and let us also denote

$$
\tilde{g}\left(z_{i}\right)=\mathbf{E}_{x_{H}} \mathbf{E}_{z \sim \mathcal{N}^{M}}\left[\operatorname{sign}\left(w_{H} \cdot x_{H}+\left(\sum_{i=1}^{M} z_{i}\right) / \sqrt{M}\right)\right] .
$$

Since the tail of $g$ is $1 / \sqrt{M}$-regular Lemma 59 implies that

$$
\sum_{i=1}^{M}\left(\widehat{g}\left(z_{i}\right)-\tilde{g}\left(z_{i}\right)\right)^{2}=O\left(M^{-1 / 12}\right)
$$

and by Fact 20

$$
\left|\sum_{i=1}^{M}\left(\widehat{g}\left(z_{i}\right)\right)^{2}-\sum_{i=1}^{M}\left(\tilde{g}\left(z_{i}\right)\right)^{2}\right|=O\left(M^{-1 / 24}\right) .
$$

Since $\widehat{F}(y)=\mathbf{E}_{x_{H}} \mathbf{E}_{y \sim \mathcal{N}}\left[\operatorname{sign}\left(w_{H} \cdot x_{H}+y\right)\right]$, arguments identical to those of Lemma 63 give us

$$
\sum_{i=1}^{M}\left(\tilde{g}\left(z_{i}\right)\right)^{2}=(\widehat{F}(y))^{2}
$$

This completes the proof. 


\section{Proof of Theorem 5: An approximation algorithm for $T(\mathbb{S})$}

In this section we prove Theorem 5 (restated below):

Theorem 5. There is an algorithm that, on input an accuracy parameter $\epsilon>0$, runs in time $2^{\text {poly }(1 / \epsilon)}$ and outputs a value $\Gamma_{\epsilon}$ such that

$$
\mathbf{T}(\mathbb{S}) \leq \Gamma_{\epsilon} \leq \mathbf{T}(\mathbb{S})+\epsilon
$$

The main structural result required to prove Theorem 5 is the following theorem (recall that $\mathbb{S}^{n-1}$ denotes the unit sphere in $\mathbb{R}^{n}$, i.e. $\left.\mathbb{S}^{n-1}=\left\{x \in \mathbb{R}^{n}:\|x\|_{2}=1\right\}\right)$ :

Theorem 69. For any $\epsilon>0$, there is a value $K_{\epsilon}=\operatorname{poly}(1 / \epsilon)$ such that for any $n \in \mathbb{N}$,

$$
\mathbf{T}\left(\mathbb{S}^{n-1}\right) \leq \mathbf{T}\left(\mathbb{S}^{K_{\epsilon}-1}\right) \leq \mathbf{T}\left(\mathbb{S}^{n-1}\right)+\epsilon .
$$

As a corollary, we have $\mathbf{T}(\mathbb{S}) \leq \mathbf{T}\left(\mathbb{S}^{K_{\epsilon}-1}\right) \leq \mathbf{T}(\mathbb{S})+\epsilon$.

Theorem 69 implies that to compute $\mathbf{T}(\mathbb{S})$ up to accuracy $\epsilon$, it suffices to compute $\mathbf{T}\left(\mathbb{S}^{K_{\epsilon}-1}\right)$; i.e., we need to compute $\inf _{w \in \mathbb{S}^{K_{\epsilon}-1}} \mathbf{T}(w)$. While $\mathbb{S}^{K_{\epsilon}-1}$ is a finite-dimensional object, it is an (uncountably) infinite set and hence it is not immediately obvious how to compute $\inf _{w \in \mathbb{S} K_{\epsilon}-1} \mathbf{T}(w)$. The next lemma says that this can indeed be computed in time $2^{\widetilde{O}\left(K_{\epsilon}^{2}\right)}$.

Lemma 70. For any $m \in \mathbb{N}, \mathbf{T}\left(\mathbb{S}^{m-1}\right)$ can be computed exactly in time $2^{\tilde{O}\left(m^{2}\right)}$.

Theorem 5 follows by combining Theorem 69 and Lemma 70

\subsection{Proof of Theorem 69}

Proof of Theorem 69 Let $w \in \mathbb{S}^{n-1}$. For $\epsilon>0$, we will prove that there exists a value $K_{\epsilon}=O\left(1 / \epsilon^{3}\right)$ and $v \in \mathbb{S}^{K_{\epsilon}-1}$ such that $|\mathbf{T}(v)-\mathbf{T}(w)| \leq \epsilon$. Clearly, the upper bound on $\mathbf{T}\left(\mathbb{S}^{K_{\epsilon}-1}\right)$ in Theorem 69 follows from this. The lower bound on $\mathbf{T}\left(\mathbb{S}^{K_{\epsilon}-1}\right)$ is obvious.

To prove the existence of vector $v \in \mathbb{S}^{K_{\epsilon}-1}$, we begin by considering the $\eta$-critical index of $w$ for $\eta=\epsilon / 64$. We also let $K=C \cdot t / \eta^{2} \cdot \log (t / \eta)$ where $t$ will be chosen later to be $O(\log (1 / \eta))$ and $C$ to be a sufficiently large constant. Clearly, for this choice of $\eta$ and $t$, we have that $K=O\left(1 / \epsilon^{3}\right)$. The next two claims show that whether $c(w, \eta)$, the $\eta$-critical index of $w$, is larger or smaller than $K$, the desired vector $v$ exists in either case.

Claim 71. Let $w \in \mathbb{S}^{n-1}$ be such that $c(w, \eta)>K$. Then there is a vector $v \in \mathbb{S}^{K}$ such that $\mid \mathbf{T}(v)-$ $\mathbf{T}(w) \mid \leq \eta$.

Claim 72. Let $w \in \mathbb{S}^{n-1}$ be such that $c(w, \eta) \leq K$. Then there exists $v \in \mathbb{S}^{K+\lambda(\eta)-1}$ such that $\mid \mathbf{T}(v)-$ $\mathbf{T}(w) \mid \leq 8 \eta$, where $\lambda(\eta)=4 / \eta^{2}$.

In both Claim 71 and Claim 72, the final vector $v$ is at most $K+\lambda(\eta) \leq O\left(1 / \epsilon^{3}\right)$-dimensional. Hence Theorem 69 follows by choosing $K_{\epsilon}=O\left(1 / \epsilon^{3}\right)$.

We start by proving Claim 71. We will require the following anti-concentration lemma from [OS11]:

Lemma 73. (Theorem 4.2 in OS11]) Let $w \in \mathbb{S}^{n-1}, 0<\eta<1 / 2, t>1$ and let $K$ be defined (in terms of $t$ and $\eta)$ as above. If $c(w, \eta)>K$, then for any $w_{0} \in \mathbb{R}$, we have

$$
\mathbf{P r}_{x \in\{-1,1\}^{n}}\left[\left|\sum_{i=1}^{n} w_{i} x_{i}-w_{0}\right| \leq \sqrt{t} \cdot \sigma_{K}\right] \leq 2^{-t}
$$

where $\sigma_{K}=\left\|w^{(K)}\right\|_{2}=\sqrt{\sum_{j>K} w_{j}^{2}}$. 
Remark 74. Lemma 73 as stated in [OS11] has the probability bounded by $O\left(2^{-t}\right)$. However, by making $C$ large enough, it is obvious that the probability can be made $2^{-t}$.

Proof of Claim 71 Choose a specific $w_{0}$ (we will fix it later). By Lemma 73, we have that,

$$
\mathbf{P r}_{x \in\{-1,1\}^{n}}\left[\left|\sum_{i=1}^{n} w_{i} x_{i}-w_{0}\right| \leq \sqrt{t} \cdot \sigma_{K}\right] \leq 2^{-t}
$$

Note that $\sum_{i=1}^{n} w_{i} x_{i}=\sum_{i \leq K} w_{i} x_{i}+\sum_{i>K} w_{i} x_{i}$. Let $w_{T}$ denote the "tail weight vector" $w_{T}=\left(w_{K+1}, \ldots, w_{n}\right)$. Since $\left\|w_{T}\right\|_{2}=\sigma_{K}$, by Hoeffding's inequality (Theorem 15), we have that

$$
\operatorname{Pr}_{x \in\{-1,1\}^{n-K}}\left[\left|w_{T} \cdot x_{T}\right|>\frac{1}{2} \cdot \sqrt{\frac{t}{2}} \cdot \sigma_{K}\right] \leq 2^{\frac{-t}{8}} .
$$

Define the set $A_{\text {good, } w_{0}}$ as follows :

$$
A_{\text {good }, w_{0}}:=\left\{x \in\{-1,1\}^{n}:\left|\sum_{i=1}^{n} w_{i} x_{i}-w_{0}\right| \geq \sqrt{t} \cdot \sigma_{K} \text { and }\left|\sum_{i>K} w_{i} x_{i}\right| \leq \sqrt{\frac{t}{8}} \cdot \sigma_{K}\right\}
$$

We next make a couple of observations about the set $A_{\text {good, } w_{0}}$. The first is that combining (17) and (18), we get that $\operatorname{Pr}_{x \in\{-1,1\}^{n}}\left[x \notin A_{\text {good, } w_{0}}\right] \leq 2^{\frac{-t}{8}}+2^{-t}$. Second, for every $x \in A_{\text {good, } w_{0}}$, we have

$$
\left|\sum_{i=1}^{K} w_{i} x_{i}-w_{0}\right| \geq\left|\sum_{i=1}^{n} w_{i} x_{i}-w_{0}\right|-\left|\sum_{i>K} w_{i} x_{i}\right| \geq \sqrt{t} \cdot \sigma_{K} \cdot\left(1-\frac{1}{2 \sqrt{2}}\right) \geq \sqrt{t} \cdot \sigma_{K} \cdot \frac{3}{5} .
$$

Hence for every $x \in A_{\text {good, } w_{0}}$, we have

$$
\mathbf{1}_{\sum_{i=1}^{n} w_{i} x_{i} \leq w_{0}}=\mathbf{1}_{\sum_{i=1}^{K} w_{i} x_{i} \leq w_{0}} .
$$

Now, consider the vector $v^{\prime} \in \mathbb{S}^{n}$ defined as follows:

- $v_{i}^{\prime}=w_{i}$ for $1 \leq i \leq K$;

- $v_{K+1}^{\prime}=\sigma_{K}$; and

- $v_{j}^{\prime}=0$ for $j>K+1$.

Note that for every $x \in\{-1,1\}^{n}$ (and hence for every $x \in A_{\text {good, } w_{0}}$ ), we have $\sum_{i=1}^{K} w_{i} x_{i}=\sum_{i=1}^{K} v_{i}^{\prime} x_{i}$. Recalling that

$$
\left|\sum_{i=1}^{K} v_{i}^{\prime} x_{i}-w_{0}\right|=\left|\sum_{i=1}^{K} w_{i} x_{i}-w_{0}\right| \geq \sqrt{t} \cdot \sigma_{K} \cdot \frac{3}{5} \quad \text { for } x \in A_{\text {good }, w_{0}}
$$

and that all $x \in\{-1,1\}^{n}$ satisfy $\left|\sum_{i>K} v_{i}^{\prime} x_{i}\right| \leq \sigma_{K}$, for $t$ sufficiently large we get that every $x \in A_{\text {good, } w_{0}}$ satisfies

$$
{ }^{\mathbf{1}_{\sum_{i=1}^{n} v_{i}^{\prime} x_{i} \leq w_{0}}}=\mathbf{1}_{\sum_{i=1}^{K} v_{i}^{\prime} x_{i} \leq w_{0}} .
$$

Thus, for $x \in A_{\text {good, } w_{0}}$, we have that all four events coincide:

$$
{ }^{\mathbf{1}_{\sum_{i=1}^{n} v_{i}^{\prime} x_{i} \leq w_{0}}}=\mathbf{1}_{\sum_{i=1}^{K} v_{i}^{\prime} x_{i} \leq w_{0}}=\mathbf{1}_{\sum_{i=1}^{K} w_{i} x_{i} \leq w_{0}}=\mathbf{1}_{\sum_{i=1}^{n} w_{i} x_{i} \leq w_{0}} .
$$


Likewise, we also get that for $x \in A_{\text {good, } w_{0}}$,

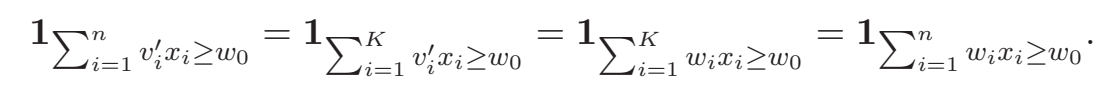

Now, let $S=A_{\text {good, } 1} \cap A_{\text {good, }-1}$. We then get that for $x \in S$,

$$
\mathbf{1}_{\sum_{i=1}^{n} v_{i}^{\prime} x_{i} \in[-1,1]}=\mathbf{1}_{\sum_{i=1}^{n} w_{i} x_{i} \in[-1,1]} .
$$

Since $\operatorname{Pr}_{x \in\{-1,1\}^{n}}\left[x \notin A_{\text {good }, w_{0}}\right] \leq 2^{\frac{-t}{8}}+2^{-t}$ for $w_{0} \in\{-1,1\}$, as a result we have $\operatorname{Pr}[x \notin S] \leq$ $2 \cdot\left(2^{-t / 8}+2^{-t}\right)$. Taking $t=8 \log (16 / \eta)$, we get that $\operatorname{Pr}[x \notin S] \leq \eta / 4$. This implies that

$$
\left|\mathbf{P r}_{x \in\{-1,1\}^{n}}\left[\left|\sum_{i=1}^{n} w_{i} x_{i}\right| \leq 1\right]-\mathbf{P r}_{x \in\{-1,1\}^{n}}\left[\left|\sum_{i=1}^{n} v_{i}^{\prime} x_{i}\right| \leq 1\right]\right| \leq \eta / 4
$$

Since the final $n-K-1$ coordinates of $v^{\prime}$ are zero, if we simply truncate $v^{\prime}$ to the first $K+1$ coordinates, we get a vector $v \in \mathbb{S}^{K}$ such that

$$
\left|\mathbf{P r}_{x \in\{-1,1\}^{n}}\left[\left|\sum_{i=1}^{n} w_{i} x_{i}\right| \leq 1\right]-\mathbf{P r}_{x \in\{-1,1\}^{K+1}}\left[\left|\sum_{i=1}^{K+1} v_{i} x_{i}\right| \leq 1\right]\right| \leq \eta / 4
$$

and Claim 71 is proved.

We next move to the proof of Claim 72 For that, we will need the following key proposition.

Proposition 75. Let $w, u \in \mathbb{S}^{n-1}$ be such that $w_{i}=u_{i}$ for $1 \leq i \leq K$. Suppose moreover that $w^{(K)} \stackrel{\text { def }}{=}$ $\left(w_{K+1}, \ldots, w_{n}\right)$ and $u^{(K)} \stackrel{\text { def }}{=}\left(u_{K+1}, \ldots, u_{n}\right)$ are both $\eta$-regular. Then, for any $w_{0} \in \mathbb{R}$, we have that

$$
\left|\mathbf{P r}_{x \in\{-1,1\}^{n}}\left[w \cdot x \leq w_{0}\right]-\operatorname{Pr}_{x \in\{-1,1\}^{n}}\left[u \cdot x \leq w_{0}\right]\right| \leq 4 \eta
$$

and

$$
\left|\mathbf{P r}_{x \in\{-1,1\}^{n}}\left[w \cdot x \geq w_{0}\right]-\mathbf{P r}_{x \in\{-1,1\}^{n}}\left[u \cdot x \geq w_{0}\right]\right| \leq 4 \eta .
$$

Proof. Consider any fixed setting of variables $x_{1}, \ldots, x_{K} \in\{-1,1\}$. Note that $\sum_{i=1}^{n} w_{i} x_{i}=\sum_{i \leq K} w_{i} x_{i}+$ $\sum_{i>K} w_{i} x_{i}$. We have

$$
\operatorname{Pr}_{x^{(K)} \in\{-1,1\}^{n-K}}\left[\sum_{i=1}^{n} w_{i} x_{i} \leq w_{0}\right]=\operatorname{Pr}_{x^{(K)} \in\{-1,1\}^{n-K}}\left[\sum_{i>K} w_{i} x_{i} \leq w_{0}-\sum_{i \leq K} w_{i} x_{i}\right] .
$$

However, as $w^{(K)}$ is $\eta$-regular, by Theorem 16 , we get

$$
\begin{aligned}
& \mid \operatorname{Pr}_{x^{(K)} \in\{-1,1\}^{n-K}}\left[\sum_{i>K} w_{i} x_{i} \leq w_{0}-\sum_{i \leq K} w_{i} x_{i}\right] \\
& -\operatorname{Pr}\left[\mathcal{N}\left(0,\left\|w^{(K)}\right\|\right) \leq w_{0}-\sum_{i \leq K} w_{i} x_{i}\right] \mid \leq 2 \eta .
\end{aligned}
$$

Likewise, we have

$$
\begin{gathered}
\mid \operatorname{Pr}_{x^{(K)} \in\{-1,1\}^{n-K}}\left[\sum_{i>K} u_{i} x_{i} \leq w_{0}-\sum_{i \leq K} u_{i} x_{i}\right] \\
-\operatorname{Pr}\left[\mathcal{N}\left(0,\left\|u^{(K)}\right\|\right) \leq w_{0}-\sum_{i \leq K} u_{i} x_{i}\right] \mid \leq 2 \eta
\end{gathered}
$$


As $w_{i}=u_{i}$ for $1 \leq i \leq K$, we get that

$$
\begin{aligned}
& \mid \operatorname{Pr}_{x^{(K)} \in\{-1,1\}^{n-K}}\left[\sum_{i>K} u_{i} x_{i} \leq w_{0}-\sum_{i \leq K} u_{i} x_{i}\right] \\
& -\mathbf{P r}_{x^{(K)} \in\{-1,1\}^{n-K}}\left[\sum_{i>K} w_{i} x_{i} \leq w_{0}-\sum_{i \leq K} w_{i} x_{i}\right] \mid \leq 4 \eta .
\end{aligned}
$$

As the above equation is true for any setting of $x_{1}, \ldots, x_{K}$, we get that

$$
\left|\operatorname{Pr}_{x \in\{-1,1\}^{n}}\left[w \cdot x \leq w_{0}\right]-\operatorname{Pr}_{x \in\{-1,1\}^{n}}\left[u \cdot x \leq w_{0}\right]\right| \leq 4 \eta
$$

The second part of the proposition follows in exactly the same way.

Proof of Claim 72 Let $w=\left(w_{1}, \ldots, w_{K}, \ldots, w_{n}\right)$ where $K^{\prime} \leq K$ is the $\eta$-critical index of $w$. Construct a new vector $v^{\prime}$ such that $v_{i}^{\prime}=w_{i}$ for $1 \leq i \leq K^{\prime}$. For $1 \leq j \leq \lambda(\eta)=4 / \eta^{2}$, we let $v_{i+j}^{\prime}=(\eta / 2) \cdot\left\|w^{\left(K^{\prime}\right)}\right\|$, where as before $w^{\left(K^{\prime}\right)}$ denotes the $\left(n-K^{\prime}\right)$-dimensional vector $\left(w_{K^{\prime}+1}, \ldots, w_{n}\right)$. For $j>\lambda(\eta)$, we define $v_{i+j}^{\prime}=0$.

It is clear that $v^{\prime} \in \mathbb{S}^{n}$ and that $v^{\prime\left(K^{\prime}\right)}$ is $\eta$-regular. By Proposition 75, we have

$$
\left|\mathbf{P r}_{x \in\{-1,1\}^{n}}[w \cdot x \leq 1]-\mathbf{P r}_{x \in\{-1,1\}^{n}}\left[v^{\prime} \cdot x \leq 1\right]\right| \leq 4 \eta
$$

and

$$
\left|\mathbf{P r}_{x \in\{-1,1\}^{n}}[w \cdot x \geq-1]-\mathbf{P r}_{x \in\{-1,1\}^{n}}\left[v^{\prime} \cdot x \geq-1\right]\right| \leq 4 \eta
$$

Combining these two, we get

$$
\left|\mathbf{P r}_{x \in\{-1,1\}^{n}}[|w \cdot x| \leq 1]-\mathbf{P r}_{x \in\{-1,1\}^{n}}\left[\left|v^{\prime} \cdot x\right| \leq 1\right]\right| \leq 8 \eta
$$

As all the coordinates of $v^{\prime}$ beyond the first $K^{\prime}+\lambda(\eta)$ coordinates are zero, if we truncate $v^{\prime}$ to its first $K^{\prime}+\lambda(\eta)$ coordinates, we get $v \in \mathbb{S}^{K^{\prime}+\lambda(\eta)-1}$ such that

$$
\left|\operatorname{Pr}_{x \in\{-1,1\}^{n}}[|w \cdot x| \leq 1]-\operatorname{Pr}_{x \in\{-1,1\}^{K^{\prime}+\lambda(\eta)}}[|v \cdot x| \leq 1]\right| \leq 8 \eta
$$

and Claim 72 is proved.

\subsection{Proof of Lemma 70}

The proof of Lemma 70 that we give below is based on the decidability of the existential theory of the reals. We believe that it may be possible to prove this lemma without invoking the existential theory of the reals, by combining perturbation-based arguments with convex programming. However, carefully formalizing such arguments is a potentially involved process, so we have given (what seemed to us to be) a more concise proof, using the existential theory of reals, below.

Proof of Lemma 70 We use the following result due to Renegar [Ren88].

Theorem 76. [Ren88] There is an algorithm $\mathcal{A}_{\text {Ren }}$ which, given a set of real polynomials $p_{1}, \ldots, p_{m}$ : $\mathbb{R}^{n} \rightarrow \mathbb{R}$ and $q_{1}, \ldots, q_{k}: \mathbb{R}^{n} \rightarrow \mathbb{R}$ with rational coefficients, decides whether there exists an $x \in \mathbb{R}^{n}$ such that

- $\forall i \in[m], p_{i}(x) \geq 0$, and 
- $\forall i \in[k], q_{i}(x)>0$.

If the bit length of all coefficients in all polynomials is at most $L$ and the maximum degree of any polynomial is at most $d$, then the running time of $\mathcal{A}_{\text {Ren }}$ is $L^{O(1)} \cdot((m+k) \cdot d)^{O(n)}$.

The following is an obvious corollary of the above theorem :

Corollary 77. There is an algorithm which, given a set $S \subset\{-1,1\}^{m}$, decides whether there exists a vector $w \in \mathbb{S}^{m-1}$ such that every $x \in S$ has $|w \cdot x|>1$. The algorithm runs in time $2^{O\left(m^{2}\right)}$.

Proof. Let $p_{0}: \mathbb{R}^{m} \rightarrow \mathbb{R}$ be defined as $p_{0}(w)=\sum_{i=1}^{m} w_{i}^{2}-1$. For each $x \in S$, define $q_{x}: \mathbb{R}^{m} \rightarrow \mathbb{R}$ to be $q_{x}(w)=\left(\sum_{i=1}^{m} w_{i} \cdot x_{i}\right)^{2}-1$. Consider the following set of constraints (call it $\left.\mathcal{L}\right)$ :

- $p_{0}(w) \geq 0$.

- $-p_{0}(w) \geq 0$.

- $\forall x \in S, q_{x}(w)>0$.

Clearly, this set of constraints has a solution if and only if there exists some $w \in \mathbb{S}^{m-1}$ such that $|w \cdot x|>1$ for all $x \in S$. Note that each of the polynomials in $\mathcal{L}$ is of degree 2 and all coefficients have constant-size representations. The total number of constraints in $\mathcal{L}$ is $|S|+2 \leq 2^{m}+2$. This means that $\mathcal{A}_{\text {Ren }}$ can decide the feasibility of $\mathcal{L}$ in time $2^{m \cdot O(m)}=2^{O\left(m^{2}\right)}$ which proves the claim.

Next, we define a set $S \subseteq\{-1,1\}^{m}$ to be a separable set if there exists some $w \in \mathbb{S}^{m-1}$ such that $S=\left\{x \in\{-1,1\}^{m}:|w \cdot x|>1\right\}$. The next claim says that we can enumerate over (a superset of) the set of all separable sets in time $2^{\tilde{O}\left(m^{2}\right)}$.

Claim 78. There is an algorithm which runs in time $2^{\tilde{O}\left(m^{2}\right)}$ and lists (a superset of ) all the separable sets of $\{-1,1\}^{m}$.

Proof. Consider any separable set $S \subseteq\{-1,1\}^{m}$, so there is some $w \in \mathbb{S}^{m-1}$ such that $S=\{x \in$ $\left.\{-1,1\}^{m}:|w \cdot x|>1\right\}$. Define $S_{+, w}=\left\{x \in\{-1,1\}^{m}: w \cdot x>1\right\}$. If we define $S_{+, w}^{\prime}$ to be the set obtained by negating every element of $S_{+, w}$, then it is easy to see that $S=S_{+, w} \cup S_{+, w}^{\prime}$. Thus, it suffices to enumerate over the sets $S_{+, w}$ for all choices of $w \in \mathbb{S}^{m-1}$, and output $S=S_{+, w} \cup S_{+, w}^{\prime}$.

Next, we show how to enumerate all such sets $S_{+, w}$. For this, given any $w \in \mathbb{S}^{m-1}$, we define $\alpha_{w}=$ $\inf _{x \in S_{+, w}}(w \cdot x-1)$. It is easy to see that if we define $h_{w}(x)=\operatorname{sign}(w \cdot x-1-\alpha / 2)$, then $S_{+, w}=h_{w}^{-1}(1)$. Thus, if we enumerate all possible halfspaces $h$ over $\{-1,1\}^{m}$ and list $h^{-1}(1)$ for each halfspace $h$, then all possible subsets of the form $h_{w}^{-1}(1)$ are included in this list. However, it is well known that there are $2^{O\left(m^{2}\right)}$ halfspaces $h$ over $\{-1,1\}^{m}$, and that these halfspaces can be enumerated in time $2^{O\left(m^{2} \log m\right)}$ (see e.g. [MORS10]). This finishes the proof of the claim.

Finally, our algorithm is simply the following:

- Run the algorithm in Claim 78 and arrange the sets in the output in decreasing order of their size. For each set $S$ in this list,

- Use the algorithm in Corollary 77 to decide whether there is a vector $w \in \mathbb{S}^{m-1}$ such that $|w \cdot x|>1$ for all $x \in S$.

- If there is such a vector $w$ then exit and return $1-|S| / 2^{m-1}$, else go to the next set $S$. 
The total running time of the first step of the algorithm is clearly $2^{\widetilde{O}\left(m^{2}\right)}$. Since the total number of sets in the list is $2^{\tilde{O}\left(\mathrm{~m}^{2}\right)}$ and every step in the algorithm takes time $2^{O\left(\mathrm{~m}^{2}\right)}$, hence the total running time is $2^{\widetilde{O}\left(m^{2}\right)}$.

To establish the correctness of the algorithm, fix a vector $w^{*} \in \mathbb{S}^{m-1}$ such that $\operatorname{Pr}_{x \in\{-1,1\}^{m}}\left[\left|w^{*} \cdot x\right| \leq\right.$ 1] $=\mathbf{T}\left(\mathbb{S}^{m-1}\right)$, i.e. $\mathbf{P r}_{x \in\{-1,1\}^{m}}\left[\left|w^{*} \cdot x\right| \leq 1\right] \leq \mathbf{P r}_{x \in\{-1,1\}^{m}}[|w \cdot x| \leq 1]$ for all $w \in \mathbb{S}^{m-1}$. We have that $\operatorname{Pr}_{x \in\{-1,1\}^{m}}\left[\left|w^{*} \cdot x\right|>1\right] \geq \operatorname{Pr}_{x \in\{-1,1\}^{m}}[|w \cdot x|>1]$. Since our algorithm enumerates over all sets $S \subseteq\{-1,1\}^{m}$ in the output of Claim 78 in decreasing order of their size, its correctness follows.

\section{Acknowledgements}

We thank Mihalis Yannakakis for helpful conversations.

\section{References}

[AHS12] N. Alon, H. Huang, and B. Sudakov. Nonnegative k-sums, fractional covers, and probability of small deviations. Journal of Combinatorial Theory, Series B, 102(3):784-796, 2012.

[BD12] V. Bentkus and D. Dzindzalieta. A tight Gaussian bound for weighted sums of Rademacher random variables. Technical report, preprint, 2012.

[BDD98] S. Ben-David and E. Dichterman. Learning with restricted focus of attention. Journal of Computer and System Sciences, 56(3):277-298, 1998.

[Ben04] V. Bentkus. On Hoeffding's Inequality. Annals of Probability, 32:1650-1673, 2004.

[BKS99] I. Benjamini, G. Kalai, and O. Schramm. Noise sensitivity of Boolean functions and applications to percolation. Inst. Hautes Études Sci. Publ. Math., 90:5-43, 1999.

[BTNR02] A. Ben-Tal, A. Nemirovski, and C. Roos. Robust Solutions of Uncertain Quadratic and ConicQuadratic Problems. SIAM Journal on Optimization, 13(2):535-560, 2002.

[DDFS12] A. De, I. Diakonikolas, V. Feldman, and R. Servedio. Near-optimal solutions for the Chow Parameters Problem and low-weight approximation of halfspaces. In Proc. 44th ACM Symposium on Theory of Computing (STOC), pages 729-746, 2012.

$\left[\mathrm{DGJ}^{+}\right.$10] I. Diakonikolas, P. Gopalan, R. Jaiswal, R. Servedio, and E. Viola. Bounded independence fools halfspaces. SIAM J. on Comput., 39(8):3441-3462, 2010.

[DS09] I. Diakonikolas and R. Servedio. Improved approximation of linear threshold functions. In Proc. 24th Annual IEEE Conference on Computational Complexity (CCC), pages 161-172, 2009.

[Fe168] W. Feller. An introduction to probability theory and its applications. John Wiley \& Sons, 1968.

[Fil12] Y. Filmus. Khintchine-Kahane using Fourier Analysis. Posted at http://www.cs.toronto.edu/ yuvalf/KK.pdf, 2012.

[Gar07] D.J.H. Garling. Inequalities: A journey into linear analysis. Cambridge, 2007.

[GL94] C. Gotsman and N. Linial. Spectral properties of threshold functions. Combinatorica, 14(1):3550, 1994. 
[Guy86] R. K. Guy. Any answers anent these analytical enigmas? American Math Monthly, 93:279-281, 1986.

[Haa82] U. Haagerup. The best constants in the Khintchine inequality. Studia Math., 70:231-283, 1982.

[HK92] R. Holzman and D. J. Kleitman. On the product of sign vectors and unit vectors. Combinatorica, 12(3):303-316, 1992.

[HK94] P. Hitczenko and S. Kwapień. On the Rademacher series. Probability in Banach spaces, pages 31-36, 1994. Birkhäuser, Boston, MA.

[HLNZ08] S. He, Z. Luo, J. Nie, and S. Zhang. Semidefinite Relaxation Bounds for Indefinite Homogenous Quadratic Optimization. SIAM Journal on Optimization, 19:503-523, 2008.

[Jac06] J. Jackson. Uniform-distribution learnability of noisy linear threshold functions with restricted focus of attention. In Proceedings of the Nineteenth Annual Conference on Computational Learning Theory (COLT), pages 304-318, 2006.

[KLO96] S. Kwapień, R. Latala, and K. Oleszkiewicz. Comparison of moments of sums of independent random variables and differential inequalities. J. Funct. Anal., 136:258-268, 1996.

[KSTJ99] H. König, C. Schütt, and N. Tomczak-Jaegermann. Projection constants of symmetric spaces and variants of Khintchine's inequality. J. Reine Agnew. Math., 511:1-42, 1999.

[LO94] R. Latala and K. Oleszkiewicz. On the best constant in the Khinchin-Kahane inequality. Studia Math., 109:101-104, 1994.

[MORS10] K. Matulef, R. O’Donnell, R. Rubinfeld, and R. Servedio. Testing halfspaces. SIAM J. on Comput., 39(5):2004-2047, 2010.

[MS90] S. Montgomery-Smith. The distribution of Rademacher sums. In Proceedings of the American Mathematical Society, pages 517-522, 1990.

[MT94] W. Maass and G. Turan. How fast can a threshold gate learn? In Computational Learning Theory and Natural Learning Systems: Volume I: Constraints and Prospects, pages 381-414. MIT Press, 1994.

[O’D12] R. O’Donnell. Analysis of boolean functions. Technical report, 2012. Book serialization available at http://analysisofbooleanfunctions.org/.

[Ole96] K. Oleszkiewicz. On the Stein property of Rademacher sequences. Probability and Mathematical Statistics, 16:127-130, 1996.

[Ole99] K. Oleszkiewicz. Comparison of moments via Poincaré-type inequality. Contemporary Mathematics, 234:135-148, 1999.

[OS11] R. O'Donnell and R. Servedio. The Chow Parameters Problem. SIAM J. on Comput., 40(1):165-199, 2011.

[Per04] Y. Peres. Noise stability of weighted majority, 2004. Available at http://arxiv.org/abs/math/0412377.

[Pin94] I. Pinelis. Extremal probabilistic problems and Hotelling's $t^{2}$ test under a symmetry condition. Ann. Statist., 22:357-368, 1994. 
[Pin12] I. Pinelis. An asymptotically Gaussian bound on the Rademacher tails. Electronic Journal of Probability., 17:1-22, 2012.

[Ren88] J. Renegar. A faster PSPACE algorithm for deciding the existential theory of the reals. IEEE Annual Symposium on Foundations of Computer Science, pages 291-295, 1988.

[Ser07] R. Servedio. Every linear threshold function has a low-weight approximator. Comput. Complexity, 16(2):180-209, 2007.

[So09] A. M.-C. So. Improved approximation bound for quadratic optimization problems with orthogonality constraints. In SODA, pages 1201-1209, 2009.

[Sza76] S.J. Szarek. On the best constants in the Khinchine inequality. Studia Math., 58:197-208, 1976.

[Tom87] B. Tomaszewski. A simple and elementary proof of the Khintchine inequality with the best constant. Bull. Sci. Math., 111(2):103-109, 1987. 